\title{
Type 2 Diabetes: One Disease, Many Pathways
}

Joon Ha

Arthur Sherman

Laboratory of Biological Modeling

National Institutes of Health

Bethesda, MD USA

\section{Abstract (249 words)}

Diabetes is a chronic, progressive disease that calls for longitudinal data and analysis. We introduce a longitudinal mathematical model that is capable of representing the metabolic state of an individual at any point in time during their progression from normal glucose tolerance to type 2 diabetes (T2D) over a period of years. As an application of the model, we account for the diversity of pathways typically

followed, focusing on two extreme alternatives, one that goes through impaired fasting glucose (IFG) first, and one that goes through impaired glucose tolerance (IGT) first. These two pathways are widely recognized to stem from distinct metabolic abnormalities in hepatic glucose production and peripheral glucose uptake, respectively. We confirm this but go beyond to show that IFG and IGT lie on a continuum ranging from high hepatic insulin resistance and low peripheral insulin resistance to low hepatic resistance and high peripheral resistance. We show that IFG generally incurs IGT, and IGT generally incurs IFG on the way to T2D, highlighting the difference between innate and acquired defects and the need to assess patients early to determine their underlying primary impairment and appropriately target therapy. We also consider other mechanisms, showing that IFG can result from impaired insulin secretion, that non-insulin dependent glucose uptake can also mediate or interact with these pathways, and that impaired incretin signaling can accelerate T2D progression. We consider whether hyperinsulinemia can cause insulin resistance in addition to being a response to it and suggest that this is a minor effect. 


\section{Introduction}

28

29

30

31

32

33

34

Diabetes is by definition a state of hyperglycemia, but its natural history is diverse. For example, some individuals experience fasting hyperglycemia first (Impaired Fasting Glucose, IFG), followed by hyperglycemia at the two-hour time point (2hPG) of an oral glucose tolerance test (OGTT), defined as Impaired Glucose Tolerance (IGT), and some experience these in the opposite order. Eventually, all people with diabetes will have both IFG and IGT (combined glucose impairment, CGI), so we refer to these two pathways as "IGT-first" and "IFG-first", respectively. An important implication of these observations is that the best period for determining differences in the underlying physiology of these pathways is during the pre-diabetic stage, when the phenotypes are still distinct.

Prediabetes is also the stage in which progression to type 2 diabetes (T2D) can be markedly delayed or prevented (45), and interventions can plausibly be made even more effective by targeting the specific metabolic defects of the patient. For example, IFG is generally thought to reflect insulin resistance at the liver, resulting in elevated hepatic glucose production (HGP), whereas IGT is thought to reflect peripheral insulin resistance, mainly in muscle, resulting in reduced glucose disposal. One would like to know whether using drugs that primarily affect hepatic or peripheral insulin resistance makes a material difference for patients on the IFG-first or IGT-first pathways, and whether any such benefit carries over once T2D has begun.

Moreover, diabetes is not a state that one enters and exits, like an infection, but a chronic condition that is the culmination of a series of gradual changes. Understanding of this progression is best obtained by longitudinal studies over a period of years or decades. Here we will focus on one such study, the Baltimore Longitudinal Study of Aging (BLSA) (51), which asked whether CGI is an obligatory stage 
52 between IFG and T2D and between IGT and T2D (Supplemental Fig. S1; all supplemental material is

53 available at Figshare, https://doi.org/10.6084/m9.figshare.10792412).

54

55

56

57

58

59

60

61

62

63

64

65

66

67

68

69

70

71

72

73

74

75

76

77

These features of diabetes suggest that a longitudinal mathematical model for disease progression could be a valuable adjunct to clinical studies. Here we establish such a model and demonstrate that it can distinguish hepatic and peripheral insulin resistance and can simulate the IGT-first and IFG-first pathways to T2D over a period of years. We will use the model to address the clinical questions raised by the BLSA and Perrault studies. By tracking virtual patients continuously in time, the model can interpolate between clinical observations, which are necessarily sparsely sampled, and indicate what is likely to have happened in the interim.

The model provides broader insight into the different insulin-resistance phenotypes, showing that a wide array of pathways, ranging from isolated IFG to isolated IGT to combinations of the two, can be obtained by combining different degrees of hepatic and peripheral insulin resistance. Thus, the apparent clinical diversity of individual paths comprises a set of quantitative variants within a unified process of metabolic dysfunction.

There is also diversity in the relative contributions of insulin resistance and beta-cell dysfunction to diabetes pathogenesis. This is particularly apparent in comparing diabetes risk factors in populations with a high prevalence of obesity, such as those of African descent and Native Americans, to populations in which diabetes risk is seen among lean individuals and beta-cell function is weaker, such as South and East Asians; populations of European descent tend to lie in between (46). Furthermore, beta-cell dysfunction can be subdivided into defects in first- and second-phase insulin secretion. As a first installment on this large and complex set of problems, we examine whether the loss of first-phase secretion early in pre-diabetes plays a fundamental causal role in T2D development or is just a useful marker. 
General modeling approach

81 The diabetes field is fortunate to have a strong tradition of mathematical modeling $(20,49)$. A common

82 feature of those models is that they provide snapshots in time of the metabolic state of an individual,

83 including insulin resistance and beta-cell function. These models have been used to track progression by taking a series of snapshots, but they do not contain mechanisms of progression and do not describe trajectories of progression.

87 The model developed here belongs to a different family of models that seek to explain disease progression mechanistically, rather than assess the current state. It is an extension of our previously published model of beta-cell mass and function, which successfully simulated progression to diabetes in rodents over months and humans over years (37). That model, in turn, was based on the seminal model of (75), which

91 expressed mathematically the hypothesis that beta-cell mass provides negative feedback on a slow time

92 scale to compensate for insulin resistance. If that compensation is inadequate, however, the toxic effects

93 of very high glucose overcome the stimulatory effects of moderately elevated glucose. The normal

94 homeostatic negative feedback is converted to positive feedback, leading to deterioration in glucose

95 tolerance and culminating in diabetes. This fundamental concept has been incorporated into other models that broadly agree but emphasize different details $(23,24,35,38,43,79)$. Notably, the model of (38) was

97 shown to be able to account for the progression of fasting hyperglycemia and T2D observed in the Diabetes Prevention Program (DPP)(45).

99 A third class of modeling studies has fit longitudinal data from clinical studies with non-linear mixed 100 effects statistical approaches to assess the magnitude of treatment effects on glucose, insulin and HbA1c $101(18,25)$ or, by fitting to a modified form of the model of $(75)$, on beta-cell mass and insulin sensitivity 102 (67). 
103 See (24) for further comment on the models cited here and other models, and see (54) for a perspective on 104 modeling T2D.

105 The initial wave of mechanistic longitudinal models simulated fasting or average daily glucose and were 106 therefore unable to describe post-prandial responses or responses to glucose challenges such as OGTTs 107 and IVGTTs. A recent model (24) added the capability of following daily and post-challenge glucose 108 variations and was fit to OGTT data from the DPP. It also introduced a distinction between peripheral 109 and hepatic insulin resistance in order to account for the effects of drug and lifestyle interventions on 110 these parameters.

112 We also, using a different methodology, introduce simulations of OGTTs at selected points during 113 progression, and separate representations for hepatic and peripheral insulin resistance. We use these new 114 features to differentiate progression by either 2hPG or FPG. We perform IVGTTs as well to illustrate the 115 evolution of the acute insulin response to glucose (AIRg), often used to assess beta-cell function. This 116 requires the model to simulate first and second phase secretion, which we accomplish by incorporating a 117 previously published model for insulin granule exocytosis (17).

This study focuses on mechanism and insight, rather than assessment. Instead of fitting parameters to particular data sets, we assume parameters and investigate the trajectories of glycemia and insulin secretion that result. We demonstrate that the model captures known features of diabetes pathogenesis data and provides novel insights and interpretations of the data. We conceptualize this as building a

123 factory, not a product. Once the utility of the model is established, we expect that a wide variety of 124 applications to clinical data will become possible. 
128 We briefly review the previous version of the model (37) and then describe the enhancements introduced

129 here. The enhanced version has been used to study clinical implications of differences in glucose time

130 courses during an OGTT (19), but was not documented in detail. Parameter values and details of

131 functions not given here are in the Appendix (Supplementary Material).

132

\section{Previous version of the model}

134 The core element, retained in the new version, is a glucose-insulin feedback loop, represented by two

135 differential equations adapted from the Minimal Model of Bergman and Cobelli (8), who built on models

136 going back to the 1960's $(2,11)$, as modified in (75):

137

$\frac{d G}{d t}=M E A L+H G P-\left(E_{G O}+S_{I} I\right) G$

138

$$
\frac{d I}{d t}=\frac{\beta}{V} I S R-k I
$$

140 The glucose $(G)$ equation (Eq. 1) says that $G$ increases on a time scale of hours as a result of meal influx

141 and hepatic glucose production $(H G P)$ and decreases as a result of uptake, which has both insulin-

142 independent and insulin-dependent components. The factor $S_{I}$ in the insulin-dependent term is closely

143 related to the well-known sensitivity to insulin reported by the Minimal Model. The insulin (I) equation

144 (Eq. 2) says that $I$ decreases due to removal, mainly in the liver, with rate constant $k$, and increases due to

145 secretion by beta cells, where $\beta$ is the beta-cell mass, ISR is the insulin secretion rate per unit mass, and $V$

146 is the volume of distribution. In the original version, ISR depended only on $G$, through the rate of beta-

147 cell metabolism $M$ :

$148 \quad I S R=\sigma \frac{(M+\gamma)^{k I S R}}{\alpha_{I S R}^{k l S R}+(M+\gamma)^{k I S R}}$

149 where $M$ was assumed to be a sigmoidally-increasing function of $G$ :

150

$M=\frac{G^{k M}}{\alpha_{M}^{k M}+G^{k M}}$ 
151 The parameter $\gamma$ in Eq. (3) represents the effect of K(ATP) channel density to shift the glucose

152 dependence of secretion (the triggering pathway (39)); when the channel density is low, $\gamma$ is high, and

153 shifts the dependence to the left, increasing secretion for the same level of $M$ because $\mathrm{Ca}^{2+}$ is higher.

154 Experiments in (34) showed that mouse beta cells in vitro adjust the K(ATP) channel density down in

155 response to sustained (overnight) elevated glucose. This has also been observed in vivo in humans (41,

$15642,44)$, along with evidence of reduced insulin clearance, $k,(19-21)$. For the most part, we omit this for

157 simplicity, but we consider the implications of reduced clearance towards the end of Results. This can be

158 viewed as the first line of defense through enhanced beta-cell function against insulin resistance over a

159 time scale of days (e.g. holiday overeating).

160 The value of $\gamma$ depends on glucose, which is taken into account by adding a third differential equation to

161 the system:

162

$163 \quad \frac{d \gamma}{d t}=\frac{\gamma_{\infty}(G)-\gamma}{\tau_{\gamma}}$

where $\gamma_{\infty}$ is an increasing sigmoidal function of $G$, and $\tau_{\gamma}$ is the time constant.

166 Insulin resistance that persists over longer periods (months in humans) despite reduced K(ATP) channel

167 density, is assumed to trigger a further level of compensatory increased beta-cell function via $\sigma$, the

168 maximal insulin secretion capacity (Eq. 3). This corresponds to the amplifying effects of metabolism

169 and/or modulators such as GLP-1 and ACh on the efficacy of $\mathrm{Ca}^{2+}$ to drive insulin granule exocytosis.

170 This second aspect of beta-cell functional compensation entails a fourth differential equation:

$171 \quad \frac{d \sigma}{d t}=\frac{\sigma_{\infty}(I S R, M)-\sigma}{\tau_{\sigma}}$ 
172 We assume that increased ISR (workload in the sense of (64) ) leads to an increase in $\sigma$ whereas

173 increased $M$ leads to a decrease in $\sigma$.

174 The slowest and final form of compensation for insulin resistance is increased beta-cell mass, $\beta$, which

175 develops over years in humans. We assume that $\beta$ is increased by proliferation, $P$, and decreased by

176 apoptosis, $A$. Following the data of (64), we assume that $P$ increases when ISR increases. We further

177 assume that apoptosis is largely driven by metabolic stress (e.g. through increased production of reactive

178 oxygen species) when glucose is high, so we make $A$ an increasing function of $M$ :

$179 \quad \frac{d \beta}{d t}=\frac{(P(I S R)-A(M)) \beta}{\tau_{\beta}}$

181 The parameters defining $P$ and $A$ are chosen such that modest increases in $G$ result in a net increase in $\beta$,

182 but large increases in $G$ result in a net decrease in $\beta$. As in the predecessor model (75), this leads to a

183 shift from compensation (negative feedback) to decompensation (positive feedback). In (37), we showed

184 that this can account for the threshold behavior observed in both rodents and humans, that is, nearly

185 steady $G$ followed by a sharp, essentially irreversible increase $(50,73)$. We proposed this as an

186 explanation for why prevention of T2D is much easier than reversing it once it is established. The same

187 dynamic properties carry over in this study with the model enhanced as described next.

\section{New features in the model}

Modeling glucose flux during daily meals and glucose tolerance tests

191 In the previous version of the model (37), $G$ represented average daily glucose and insulin levels in

192 response to steady glucose input. To address IFG and IGT, we need to be able to dissociate fasting

193 glucose from post-challenge glucose. The first step is to introduce variable glucose influx from meals, 
represented by the term $M E A L$ in Eq. (1). Timing of meals is standardized to 6:00 AM, 12:00 Noon, and 6:00 PM. The expression we use is:

196

$M E A L=\operatorname{meal}_{\max }\left(\frac{t^{m k}}{\alpha_{\text {meal }}+t^{m k}}\right)^{\eta} \exp (-\mu t)$

and Fig. S3 shows the glucose flux (panel A) and the resulting plasma glucose concentrations (panel B).

198

199

200

201

202

203

204

205

206

207

208

209 This is sufficient to give the typical drop and recovery of $H G P$ after a meal (Fig. S4A).

210

Less obvious, but also important, we need to account for the correlation between hepatic and peripheral

211 insulin resistance, which we do by making two of the parameters in Eq. 8, hepa $a_{\max }$ and $\alpha_{H G P}$, functions of $212 S_{I}$ :

$$
H G P=\frac{h e p a_{\max }}{\alpha_{H G P}+h e p a_{S I} I}+H G P_{b a s}
$$




$$
\alpha_{H G P}=\frac{\alpha_{b a r}}{\alpha_{k}+S_{I}}+\alpha_{s h}
$$

216 Both hepa $a_{\max }$ and $\alpha_{H G P}$ decrease with $S_{I}$, as shown in Figs. S4B, C, and the net effect is that $H G P$ 217 decreases as $S_{I}$ increases (Fig. S4D).

218 If this, or something like this, is not done, then severe peripheral resistance combined with strong compensatory insulin secretion can result in fasting hypoglycemia, which is not the typical pattern (see Fig. S5).

221 In more typical cases of progression to pre-diabetes or diabetes, the relative impairment in insulin 222 secretion would mask this effect: the level of glycemia would be reduced but hypoglycemia would not 223 result. We chose the parameters such that HGP remains normal when insulin is elevated unless there is a 224 defect in beta-cell mass or function.

225 Figure S6, E- H shows the response of HGP and glucose disposal to a simulated hyperinsulinemic, 226 euglycemic clamp, with steady-state values in good agreement with experimental data (6).

227 To represent hepatic insulin resistance over and above the component related to peripheral insulin 228 resistance, we decrease the parameter hepa $a_{S I}$ in Eq. A4, which increases HGP at any value of $I$.

229 Equations 9-11 are phenomenological expressions that achieve the goals of making $H G P$ decrease with $230 I$ and increase when insulin resistance is present, but other expressions could also possibly work. We 231 considered making the value of hepa $a_{S I}$ depend on $I$ as an alternative way to avoid hypoglycemia, but this

232 did not work well. It is also possible that glucagon, which is not in the model, would help to prevent 233 hypoglycemia (see Limitations of the study in the Discussion). 
236 To study the dynamics of glucose and insulin under glucose challenges such as meals, OGTT, and

237 IVGTT, the model needs to account for the multiple kinetic components of insulin secretion. We adapted

238 an existing model of insulin granule exocytosis (17), which was designed to capture the biphasic pattern

239 of ISR in response to a glucose step in vitro or a hyperglycemic clamp in vivo. The first phase is

240 characterized by a sharp peak of ISR during the first 10 minutes and the second phase by a steady increase

241 of ISR over the next hour. Figure S6 shows a simulated OGTT (panels A, B) and a simulated IVGTT

242 (panels, C, D) compared to experimental data.

243 These phases are mediated by progression of vesicles through a sequence of stages culminating in

244 exocytosis (fusion with the plasma membrane and release of insulin to the circulation; Fig. S2A). A large

245 reserve pool, treated as an inexhaustible reservoir, feeds the docked pool (vesicles at binding sites on the

246 plasma membrane). Once docked, vesicles are primed and enter the readily releasable pool (RRP).

247 Primed vesicles join the immediately releasable pool by becoming closely associated with voltage-

248 dependent $\mathrm{Ca}^{2+}$ channels. First-phase secretion depends mainly on the size of the RRP at basal glucose,

and second phase secretion is controlled by the rate of mobilization of vesicles to the docked pool.

250

251

252

253

254

255

256

257

258

259

260

The insulin secretion rate ISR in the first version (37) of the model (Eq. 3) is in the new version no longer a function of glucose, but is calculated as an output of the exocytosis model (Eqs. A11), ISR thus now depends on the history of exposure to $G$, as it should, not just the current value. The exocytosis model requires as input the cytosolic $\mathrm{Ca}^{2+}$ concentration, which is modeled as a sigmoidal function of the betacell metabolic rate $M\left(\right.$ Eq. A7), and the much higher $\mathrm{Ca}^{2+}$ concentration in the microdomains of $\mathrm{Ca}^{2+}$ channels, which is modeled as a function of cytosolic $\mathrm{Ca}^{2+}$ (Eq. A8). The dose response curve shift $\gamma$, previously included in Eq. 3, represents the dynamic changes in K(ATP) channel density as before, but now explicitly alters cytosolic $\mathrm{Ca}^{2+}$ at a given level of $M$ (Eq. A7). Cytosolic calcium enhances the rates of mobilization of the reserve pool to the plasma membrane (Eq. A10) and priming of docked vesicles $\left(r_{2}\right.$ in Eqs. A12), whereas vesicle fusion is primarily controlled by microdomain $\mathrm{Ca}^{2+}$. The amplifying effect 
261 of glucose (39) is incorporated as a multiplicative factor in the rate of vesicle mobilization ( $G_{F}$ in Eq. A9).

262 The effect of the incretin GLP-1and drugs that mimic it to increase insulin secretion is modeled as

263 enhancing the amplifying effect of glucose by increasing the parameter $G_{F m a x}$ in Eq. A9 and as enhancing

264 vesicle priming by increasing the parameter $r_{2}^{0}$ in Eq. A12. This is in accord with a key feature of these

265 agonists, that they have little or no effect on secretion in the absence of glucose, minimizing the risk of

266 hypoglycemia that plagues sulfonylureas. Fig. S2C shows the effect of a two-fold increase in the incretin

267 effect on insulin secretion rate during a hyperglycemic clamp, in agreement with the effect of the GLP-1

268 receptor agonist liraglutide in the RISE study (68). Modeling the effect of incretins on glucose is more

269 difficult, as it would require including their effects to inhibit glucagon secretion or promote weight loss,

270 which is not feasible in the current model.

271 For IVGTT and hyperglycemic clamp simulations, we reduce $G_{F}$ by about a factor of two to represent the

272 lack of the incretin effect on vesicle mobilization and reduce $r_{2}^{0}$ (Eq. A12) about 10-fold to represent the

273 lack of incretin effect on vesicle priming. The rate of mobilization (Eq. A11) is also assumed to be

274 proportional to the variable $\sigma$ (Eq. 6), which thus controls the magnitude of second-phase insulin

275 secretion. As a consequence, ISR implicitly includes $\sigma$ as a multiplicative factor, as in Eq. (3) of the

276 simpler, first version of the model (37), and the dynamic evolution of the maximal secretory capacity over

277 long time scales (months) is essentially equivalent.

\section{Equations for insulin sensitivity}

280 With one exception we model peripheral insulin sensitivity as an intrinsic property of the target tissues,

281 muscle, liver and adipose, determined implicitly by obesity and genetics. In the model, this makes it an

282 external, time-dependent parameter of the system, and we examine how hyperinsulinemia compensates 
283 for insulin resistance. We model insulin sensitivity, $S_{I}$, as an exponentially decreasing function of time,

284 following (75):

$285 \frac{d S_{I}}{d t}=\frac{\left(\operatorname{tar}_{S I}-S_{I}\right)}{\tau_{S I}}$

286 In Fig. 9, we consider a hypothesis under current discussion in the literature $(35,65,71,74)$, that insulin

287 resistance may be caused by hyperinsulinemia, rather than the other way around. We think it is

288 implausible that this is the sole cause of insulin resistance, and propose instead that the core component of

289 insulin resistance is due to obesity and genetics but modified by the level of insulin $I$ as a result of the

290 negative feedback generated in the insulin signaling cascade (70). We represent the effect, which we term

291 induced resistance, as follows:

$292 S_{I}=S_{I, 0} E$

293 where

$294 \quad \frac{d S_{I, 0}}{d t}=\frac{\left(\operatorname{tar}_{S I}-S_{I, 0}\right)}{\tau_{S I}}$

295 and

$296 \frac{d E}{d t}=\frac{\left(E_{\infty}(I)-E\right)}{\tau_{E}}$

297 Equation (15) says that $E$ relaxes towards $E_{\infty}$ with a time constant $\tau_{E}$, where $E_{\infty}$ is a sigmoidally

298 decreasing function of $I$,

299

$E_{\infty}(I)=\bar{E}\left[I-\frac{\left(I-I_{S h}\right)^{k E}}{\alpha_{E}{ }^{k E}+\left(I-I_{s h}\right)^{k E}}\right]+E_{b}$

several examples of which are shown in Fig. 9F. The time constant $\tau_{E}$ is set to two days so that $E$, and

301 hence $S_{I}$, will respond to sustained hyperinsulinemia, not to normal insulin fluctuations following meals 
or glucose tolerance tests. The effect is propagated into hepatic insulin resistance through the terms containing $S_{I}$. Other parameters are in the legend for Fig. 9 and Table S17.

306 Following ADA criteria, we define IFG as FPG $>100 \mathrm{mg} / \mathrm{dl}$ but $<126 \mathrm{mg} / \mathrm{dl}$, IGT as $2 \mathrm{hPG}>140 \mathrm{mg} / \mathrm{dl}$

307 but $<200 \mathrm{mg} / \mathrm{dl}$, and T2D as FPG $\geq 126 \mathrm{mg} / \mathrm{dl}$ or $2 \mathrm{hPG} \geq 200 \mathrm{mg} / \mathrm{dl}$. Combined glucose impairment

308 (CGI) is defined as co-occurrence of IFG and IGT.

311 The model equations are solved using xppaut (5) and Matlab (The Mathworks, Natick, MA). Input files

312 defining the parameters and initial conditions are available on Figshare at:

313 https://doi.org/10.6084/m9.figshare.10792412).

Results

317 In Figs. 1 - 4, we carry out longitudinal simulations over a period of five years starting in the NGT state

318 in which either peripheral insulin resistance is dominant, in which case the first stage of hyperglycemia is

319 IGT, or hepatic insulin resistance is dominant, in which case the first stage of hyperglycemia is IFG.

320 These assumptions are applied by modeling peripheral and hepatic insulin sensitivity as exponentially

321 decreasing, using Eqs. A13 and A14, respectively. The initial values and rates of decline are the same for

322 all figures, but the steady state (target) values, $\operatorname{tar}_{S I}$ and tar $_{h e p a S I}$, are varied. The simulations calculate the

323 daily responses to meals, but we plot only the results of OGTTs carried out periodically over the five-year 
324 time span by pausing the longitudinal simulation. The corresponding peak postprandial glucose is shown

325 in Fig. S7. The capacity of beta-cell function to compensate for insulin resistance is assumed to be

326 limited, except in Fig. 2. The defect consists of a right shift in $\gamma_{\infty}$ relative to Fig. 2, which can be

327 interpreted as a slight gain of function mutation in KCNJ11, the Kir6.2 component of the KATP channels.

328 Alterations in $\sigma_{\infty}$ representing a mild defect in insulin granule mobilization would have a similar effect.

329 Parameters that were varied to make the figures are listed in Table S12A in the Appendix, and the initial 330 conditions for Figs. $1-4$ are in Table S12B.

\section{IGT-first pathway}

333 Figure 1 shows a longitudinal simulation of the effects of a strong decrease in peripheral insulin

334 sensitivity $S_{I}$ (Fig. 1A) combined with a mild decrease in hepatic insulin sensitivity, hepa SI (Fig. 1B). As

335 insulin resistance progresses, 2hPG increases rapidly while FPG increases more slowly (Fig. 1C),

336 resulting in progression from normal glucose tolerance (NGT) to IGT, CGI and ultimately T2D (Fig. 1C).

337 Fasting plasma insulin (FPI) and 2-hour plasma insulin (2hPI) rise as the beta cells initially compensate

338 partially for the insulin resistance, then fall as the beta cells fail (Fig. 1D), following the classic "Starling

339 law" of the pancreas (27). The initial rise in secretion results from an increase in beta-cell sensitivity to

340 glucose (the variable $\gamma$ increases, not shown), and the decline results from a fall in the slower component

341 of beta-cell function, $\sigma$ (Fig. 1E). Beta-cell mass $(\beta)$ also rises and falls, but the variation is limited

342 because of the slowness of $\beta$, and the fall occurs only after T2D is already underway (Fig. 1F). This

343 accords with observations that beta-cell mass is elevated in insulin-resistant pre-diabetics but reduced in

344 long-standing diabetes $(15,66,69)$. 
A subtle but important point of this simulation is that insulin resistance in both the liver and peripheral tissues reaches saturation (Fig. 1A, B) well before the advent of T2D. It is rather the continuing fall in

347 beta-cell function, $\sigma$, that drives conversion to T2D. The same sequence was seen in the simulation of T2D progression in Zucker diabetic fatty rats (Fig. 6 in (37)) and in data from monkeys (10).

The fall in $\sigma$ is triggered by the hyperglycemia and glucotoxicity that follows the early loss of insulin sensitivity (Fig. 1D) but would not lead to T2D if the pre-existing capacity of beta-cell function to

351 compensate were stronger. This is illustrated by a simulation with the same degree of insulin resistance

352 as in Fig. 1, but a milder beta-cell defect, which mimics a non-diabetic subject with insulin resistance

353 (Fig. 2). FPG and 2hPG increase modestly in response to insulin resistance and reach a plateau in the

354 IGT state as $\sigma$ levels off (Fig. 2E) and plasma insulin plateaus (Fig. 2D). The limitation of the rise in

355 glucose reduces the effects of glucotoxicity and buys time for beta-cell mass to increase and stabilize the

356 IGT state (Fig. 2F).

357 Figures 1 and 2 together paint a picture in which not only is a combination of insulin resistance and

358 impaired secretion necessary for T2D, but insulin resistance develops and saturates first, and T2D

359 develops only if the beta cells fail. Some defect in beta-cell function is required even for pre-diabetes, in

360 agreement with (28). Conversely, a pre-existing defect in insulin secretion would be silent in the absence 361 of insulin resistance (Fig. S8), unless it were severe (Fig. S9).

\section{IFG-first pathway}

364 In Fig. 3 we illustrate a contrasting case to Fig. 1, dominant hepatic insulin resistance with minor 365 peripheral resistance (Fig. 3A, B); the same beta-cell function defect is assumed as in Fig. 1. Hepatic 366 insulin resistance drives FPG across the threshold for IFG, while $2 \mathrm{hPG}$ remains below the threshold for 367 IGT (Fig. 3C). After the initial threshold crossing, however, FPG and 2hPG continue to rise, and IFG 
progresses to CGI as in Fig. 1. Insulin again rises with the help of $\gamma$ (not shown) and falls when the drop in $\sigma$ becomes too great (Fig. 3E). As in the IGT-first pathway, the conversion to T2D is driven mainly by reduced beta-cell function, $\sigma$, because insulin resistance in both the liver and peripheral tissues saturates well before T2D (or even CGI) begins. Beta-cell mass again plays a minor role (Fig. 3F).

In the BLSA some subjects who had progressed from NGT to IGT went on to T2D at the next follow-up, which prompted the authors to ask whether CGI could be skipped (51). Figure 4 demonstrates that this can happen if peripheral insulin resistance is made much greater than hepatic resistance (compare Fig. 4A to Fig. 1A). Extreme loss of peripheral insulin sensitivity causes $2 \mathrm{hPG}$ to rise dramatically, while FPG remains in the normal range, converting NGT to IGT. $2 \mathrm{hPG}$ continues to deteriorate without a substantial increase in FPG, resulting in progression of IGT to T2D without passing through CGI. Eventually, FPG crosses the thresholds for IFG and T2D, but after the individual has already reached T2D based on $2 \mathrm{hPG}$.

Figures 1 and 3, respectively, consider extreme cases of peripheral insulin resistance (PIR), where $S_{I}$ dominates, and hepatic insulin resistance (HIR), where hepa $a_{S I}$ dominates . However, most people on the path to T2D will have both. A composite view of the cases of Figs. 1 and 3 together with intermediate phenotypes is given in Fig. 5, where trajectories are plotted in the FPG - 2hPG plane. The only difference among the trajectories is the degree of HIR and PIR, varied inversely from lower right to upper left; the compensatory capacity of beta-cell function is identical for all traces. Fig. 5A shows that the trajectories diverge markedly as the defects in HIR and PIR set in but converge as hyperglycemia worsens; this happens because IFG induces IGT and IGT induces IFG. Thus, all the virtual patients end up looking the same as time goes on. The latter part of the NGT stage and the early part of the IGT stage, shown expanded in Fig. 5B, are when the underlying pathologies give rise to the most distinct behavior. This is important both for designing clinical studies and for stratifying patients for treatment. The figure suggests that the slope of the trajectory from two or more OGTTs spaced suitably far apart in time could give a good indication of the future path of the patient. 
We next look more closely at the pathogenesis process as it would appear clinically by simulating OGTTs

(Fig. 6) and IVGTTs (Fig. 7) at representative times for each stage of glucose tolerance, indicated by the black circles on the time axes in Figs. 1C and 3C.

\section{Simulation of OGTTs}

Figure 6A, C shows representative glucose and insulin profiles during OGTTs at each stage of the IGT-

first pathway of Fig. 1. Insulin concentrations at the IGT stage (Fig. 6C, dotted curve) are increased compared to NGT (Fig. 6C, solid curve) but are inadequate to maintain normoglycemia at two-hours because of the decreased peripheral insulin sensitivity (Fig. 1A). In contrast, the level of fasting insulin at

401 IGT (Fig. 6C, dotted curve) is sufficient to maintain fasting glucose within the normal range because hepatic insulin resistance is relatively mild (Fig. 1B). During CGI, glucose (Fig. 6A, dashed curve) is increased at all time points compared to IGT, while the insulin level (Fig. 6C, dashed curve) is slightly diminished at the early time points (relative to glucose, however, secretion is impaired at all time points).

This indicates that even progression to CGI from IGT is mainly due to impaired beta-cell function.

411 greater than for $2 \mathrm{hPG}$, so the threshold for IFG is crossed first in Fig. 3. Even though fasting insulin at

412 the IFG stage (Fig. 6D, dotted curve) is very high, it is not enough to maintain normal FPG, because of

413 the severe hepatic insulin resistance (Fig. 3B). However, 2hPG is maintained in the normal range because

414 peripheral insulin resistance is mild (Fig. 3A). Since both peripheral and hepatic insulin sensitivity 
415

416

417

418

419

420

421

422

423

424

425

426

427

428

429

430

431

432

433

434

435

436

437

saturate before the onset of CGI (Fig. 3A, B), the decrease in insulin at all time points during the OGTT (Fig. 6D, dashed curve) due to falling beta-cell function (Fig. 3E) is the main contributor to the progression to CGI from IFG and then to T2D.

\section{Simulation of IVGTTs}

Figures $1-6$ highlight the importance of secretion defects, in the context of insulin resistance, in all the pathways to T2D, but now we break out the contributions of early vs. late secretion. First-phase secretion is widely considered a key early marker of future progress. For example, the classic paper (13) reported a cross-sectional study of IVGTTs, and showed that AIRg declines as FPG rises and is nearly gone by the time FPG reaches $115 \mathrm{mg} / \mathrm{dl}$, well below the threshold for T2D. This supports the use of AIRg, and by implication first-phase insulin secretion, as an early marker for T2D. We now show that the model can reproduce the negative correlation between FPG and AIRg, but that rising FPG is not necessarily the sole or proximal cause of the decline in AIRg.

Figures 7A, B show simulations of the insulin responses during IVGTTs performed during the IGT-first and IFG-first pathways, respectively. AIRg is blunted and then vanishes in both pathways as FPG rises, as found in (13), but $2 \mathrm{hPG}$ also rises at the same time, albeit to different degrees relative to FPG in the two pathways. The decline of AIRg is more rapid than seen experimentally along both pathways (14), but the main point is that it results from the decline of RRP size (Figs. 7C, D), which is more fundamental than the level of glycemia, as the next two paragraphs explain.

RRP size is controlled by two factors, the rate of secretion, which determines the rate of vesicle efflux from the RRP and the rate of vesicle influx into the RRP from the docked pool. High FPG increases the rate of efflux in the basal state, so the RRP will already be depleted when the IVGTT commences, and AIRg will consequently be reduced. The rate of influx depends on the size of the docked pool and the 
rate of priming of docked vesicles. The rate of priming does not vary much in our simulations, but the

439 size of the docked pool does, depending mainly on the rate of docking, which is proportional to one of our

440 beta-cell function variables, $\sigma$. Although modest increases in glucose stimulate vesicle docking, larger increases cause glucose toxicity, which reduces $\sigma$ and hence docking.

Because $\sigma$ is slow, it responds to the average daily glucose, including the contributions of both fasting

\section{Targeted Drug Therapy}

455 With the previous, simpler model (37) we showed that NGT and T2D were bistable states separated by a 
461 Figures 8A, B contrast two drug therapies targeted to peripheral vs. hepatic insulin resistance in patients

462 in the early stages of diabetes. Figure 8A shows glucose for a patient on the IGT-first pathway with

463 dominant peripheral insulin resistance as in Fig. 1, in the absence of therapy (control, black curves) or in

464 response to a high dose of a drug targeted to peripheral insulin resistance (dashed curves) or a drug

465 targeted to hepatic insulin resistance (dotted). The high dose of the appropriately targeted drug only transiently improves FPG and 2hPG and ultimately fails to reverse T2D. Nonetheless, it is more effective at delaying progression than the mistargeted drug.

Figure 8B represents the complementary case, a patient on the IFG-first pathway with dominant hepatic insulin resistance, as in Fig. 3. The same treatments are applied, and, as in panel A, both drugs only transiently improve FPG and $2 \mathrm{hPG}$, but the appropriately targeted drug is more effective at delaying

471 progression.

Figures $8 \mathrm{C}$, D show the same drug therapies and progression pathways as in panels $\mathrm{A}$ and $\mathrm{B}$, but with the

473 drugs applied before the onset of diabetes. A low dose of a drug targeted to the patient's specific insulin

474 resistance pathology is in both cases now able to prevent progression to diabetes, while a low dose of a

475 mistargeted drug only delays progression. These examples suggest that the effectiveness of drug therapy

476 depends on both early initiation of treatment and detection of the major metabolic abnormality.

477 The study in (24) found that it was necessary to assume that the efficacy of treatment wanes with time in

478 order to fit the data from lifestyle and drug interventions in the DPP. Here we have shown that even if the

479 efficacy of treatment is maintained, the intrinsic dynamics of progressive beta-cell dysfunction can cause

480 treatment to fail.

481 Caution should be used in interpreting the simulated treatments in Fig. 8 in terms of currently used drugs.

482 For example, in the DREAM study (29) it was found that rosiglitazone was effective in cases of isolated

483 IFG (IIFG), that is IFG in the absence of IGT. Although rosiglitazone is often thought of as primarily 
improving peripheral insulin sensitivity, it also improves hepatic insulin sensitivity (81). In addition,

IIFG does not necessarily imply pure hepatic insulin resistance. For example, a person with FPG $=115$ and $2 \mathrm{hPG}=135$ would be classified as IIFG but may have significant peripheral insulin resistance. Most individuals with pre-diabetes likely have a mixture of peripheral and hepatic insulin resistance.

\section{Hepatic Insulin Clearance}

489

490

491

492

493

494

495

496

497

Another way to generate a compensatory increase in insulin is to reduce clearance. There is considerable evidence that insulin clearance is positively correlated with peripheral insulin sensitivity $(3,63)$, but the direction of causation is not clear. If clearance is reduced secondary to reduced overall insulin sensitivity, then the increased insulin would contribute to the compensatory response, along with increased secretion. However, it has been suggested that the increased insulin resulting from reduced clearance may also contribute to insulin resistance (61). In Fig. 9, we explore the consequences of reduced clearance, assuming that the increased insulin reduces $S_{I}$ is reduced by varying degrees as $I$ is increased, as described in Methods. We assume for simplicity that clearance itself is constant throughout each simulation rather than adapting to glucose, insulin or insulin sensitivity. The control case, shown in black, is equivalent to Fig. 1, an example of an IGT-first pathway to T2D. If clearance is reduced, and a modest degree of additional insulin resistance is assumed (green curve in panel F), such that the $S_{I}$ is only slightly lower than the control case (panel A), then the progression to T2D is delayed (compare black and green curves in panel C). (The smallness of the difference in $S_{I}$ is due to the fact that glucose is elevated to levels where the induced effect is triggered only for a brief period of each day.) Note that $\sigma$ is slightly larger (green vs. black, panel E), indicating that reducing clearance partially spared beta-cell function.

If the effect of insulin to induce resistance is made larger (blue curves), the beneficial effect of higher $I$ due to reduced clearance and the harmful effect of reduced insulin sensitivity approximately cancel out. Finally, if the induced resistance effect is made extreme, such that even a slight increase in $I$ reduces $S_{I}$ in half (red curves), the net effect is somewhat accelerated progression. We conclude that induced insulin 
508

509

510

511

512

513

514

515

516

517

518

519

520

521

522

523

524

525

526

527

528

529

resistance makes at best a modest contribution to the progression to T2D. If an even more extreme degree of induced resistance is assumed, such that even a slight increase in $I$ reduces $S_{I}$ to near 0 , then it is possible for frictional resistance to be the main driver of T2D, but we consider such a scenario implausible.

\section{Non-Insulin-Mediated Glucose Uptake}

We have focused up to this point on the contributions of hepatic and peripheral insulin resistance in driving the IFG-first and IGT-first pathways. However, non-insulin-mediated glucose uptake, also known as glucose effectiveness, is comparable in magnitude to insulin-dependent uptake (9) and has been found to be impaired in T2D (30). It is thus a potential target of drug therapy. We model this by varying the model parameter $E_{G 0}$ (similar to $S_{g}$ in Minimal Model analysis) in Eq. 1. In Fig. S10 we demonstrate that cutting glucose effectiveness in half can result in T2D in someone otherwise not at risk, such as the case shown in Fig. 2. Conversely, in Fig. S11 we show that doubling glucose effectiveness can prevent T2D in someone otherwise predisposed, such as the case shown in Fig. 1.

\section{Discussion}

In recognition of the fundamental character of type 2 diabetes (T2D) as a progressive disease that develops over many years, we have established a longitudinal model for its pathogenesis. We follow in the footsteps of other longitudinal models $(23,24,75)$ but offer new clinical applications and insights.

We apply the model to analyze the diverse presentation of hyperglycemia, which may manifest first in fasting glucose (IFG-first pathway) or two-hour glucose during an OGTT (IGT-first pathway). To carry out this program, we modified the representation for beta-cell response to insulin resistance in the first 
version of the model (37), enhancing it to differentiate between hepatic and peripheral insulin resistance.

The simulations show that heterogeneity in the degree of the two forms of insulin resistance can account for a wide variety of observed patterns, supporting the idea of T2D as a unitary disease with quantitative variants. We have focused on extreme cases to highlight the differences (e.g. Fig. 1 vs. Fig. 3), but the family of trajectories in the FPG-2hPG plane (Fig. 5) shows that these lie on a continuum. Figure 5 also highlights that differences in insulin resistance phenotype are most evident in the late NGT and early prediabetes stages, which are thus most amenable to differential phenotyping and therapeutic stratification.

We have incorporated a description of insulin granule dynamics sufficient to account for both first- and second-phase insulin secretion. This made it possible to simulate OGTT and IVGTT time courses and show how they are transformed systematically during progression along the two canonical pathways to diabetes (Figs. 6, 7). We also showed that sufficiently strong beta-cell function can prevent T2D even when insulin resistance is severe, allowing individuals to maintain a permanent state of IGT (Fig. 2) or even revert from IGT to NGT (not shown). Conversely, sufficient insulin sensitivity can prevent T2D even when beta-cell function is somewhat impaired (37). As discussed below under Secretion defects, a fuller treatment of the differences in the balance of insulin secretion and insulin action defects is needed to account fully for the diverse patterns of T2D progression.

We summarize below the specific lessons learned and questions answered by this study and give a preview of the clinical applications we anticipate for the model.

\section{Questions raised in the BLSA and other studies}

The BLSA study (51) asked whether subjects who enter the IGT state necessarily pass through CGI on the way to T2D. The model suggests (Fig. 4) that this is not the case, but skipping CGI happens only if the peripheral insulin resistance is markedly greater than hepatic insulin resistance, which may be relevant for adolescents, who experience extreme obesity and insulin resistance. An intermediate possibility predicted 
555 by the model is a short, but not absent, interval of CGI that could escape detection if the follow-up

556 interval is too long.

557 A parallel question is whether individuals can go directly from IFG to T2D without passing through CGI.

558 This is harder than going directly from IGT to T2D because 2hPG is much more labile than FPG; it is

559 difficult to get an increase in FPG sufficient to cross the threshold for T2D $(125 \mathrm{mg} / \mathrm{dl})$ without at the

560 same time having $2 \mathrm{hPG}$ cross the threshold for IGT $(140 \mathrm{mg} / \mathrm{dl})$. Indeed, we have not been able to

561 simulate this scenario just by choosing an appropriate mixture of hepatic and peripheral insulin sensitivity

562 using the other parameters as in Figs. $1-4$, but model simulations (not shown) predict that it can happen

563 if a more severe beta-cell defect (in $\gamma_{\infty}$, Eq. A15) is assumed.

564 The BLSA study also asked whether individuals can pass directly from NGT to T2D without passing

565 through any pre-diabetic state. Because glucose is in quasi-steady state with the much slower variables

566 representing beta-cell mass, beta-cell function and insulin sensitivity, this is not possible in the model

567 unless one of those slow variables undergoes a catastrophic, virtually discontinuous, change.

568 Pancreatectomy would be an example of this, but even type 1 diabetes, triggered by a rapid fall in beta-

569 cell mass, has a distinct prediabetes phase. The cases observed in the BLSA in which subjects were NGT

570 at baseline and T2D at first follow-up most likely reflected rapid progression or a long gap between visits.

571 Similarly, the model simulations indicate that it is unlikely for individuals to go from NGT to CGI

572 without passing through IFG or IGT.

573 The BLSA reported that IFG is generally followed by IGT and IGT is generally followed by IFG, and the

574 model suggests that each state induces the other. This happens because the initial rise in glucose during

575 IFG impairs beta-cell function, which causes $2 \mathrm{hPG}$ to rise, and vice versa. Another study raised the

576 question of whether CGI is a progressed state of IFG (57); the model simulations together with the BLSA

577 data show that CGI may instead be a progressed state of IGT, to which IFG has been added. 
578

579

580

581

582

583

584

585

586

587

588

589

590

591

592

593

594

595

596

597

598

599

600

One can also ask whether crossing the threshold for FPG or $2 \mathrm{hPG}$ of pre-diabetes predicts whether T2D will be reached by crossing the corresponding threshold. In Fig. 1, IGT is followed by T2D diagnosed through $2 \mathrm{hPG}$, and further simulations with the model (Fig. 5) suggest that this is typical. In Fig. 3, IFG is followed by T2D diagnosed through FPG, but further simulations (not shown) indicate that this may or may not be the case, depending on the degree of discrepancy between HIR and PIR and the strength of $\gamma$ to control FPG.

Both the IFG- and IGT-first pathways exhibit elevated fasting insulin. This may account for the observation that elevated fasting insulin was a better predictor of future diabetes in a prospective study than fasting glucose, which is elevated early on only in the IFG-first pathway (22); it may not be necessary to hypothesize a major causative role for high fasting insulin itself. Indeed, in the IGT-first pathway, which is more common, fasting glucose may be suppressed by the compensatory increase in beta-cell function (our variable $\sigma$ ) induced by high post-load glucose (compare Figs. 1 and 4 to Fig. 3).

The suppression of fasting glucose in the context of a predominance of peripheral insulin resistance has particular importance for pre-diabetes screening in populations that are prone to IGT but not IFG. This applies notably to people of African descent, for whom fasting glucose has markedly reduced sensitivity for detecting pre-diabetes and diabetes (77). The problem is exacerbated for Africans living in Africa, where measuring $2 \mathrm{hPG}$ with OGTTs is prohibitively expensive. The model suggests that in this and similar cases, lowering the threshold for diagnosing pre-diabetes based on fasting glucose could be a costeffective strategy. More generally, the model points to the need for population- and patient-specific thresholds for diagnosis, which may contribute to resolving current debate on whether prediabetes is a useful diagnosis (62).

\section{Insulin action defects}


601 We have varied peripheral and hepatic insulin resistance independently to study their contributions to 602 T2D progression, but in reality, they are related. Statistically, they are correlated with a coefficient of 603 about 0.7 (1). This is expected for several reasons. For one, they share major components of the insulin 604 signaling pathway. Also, there is evidence that an important determinant of hepatic insulin resistance is excess supply of free fatty acid (FFA) substrate from adipose tissue (58). Thus, insulin resistance in adipose cells would increase lipolysis and FFA flux to the liver, which would drive increased gluconeogenesis (59). On the other hand, the liver has unique roles in glucose and lipid production not shared with muscle, which may account for the fact that the correlation is imperfect.

To address hepatic insulin resistance above and beyond the component correlated with peripheral insulin resistance, we have independently varied the affinity of HGP for insulin (parameter hepa $a_{S I}$ in Eq. 9), the

611 effect of which is shown in Fig. S4. We have obtained similar results (not shown) by varying the 612 maximal rate of HGP (parameter hepa $a_{\max }$ ).

613 Although we have primarily treated insulin resistance as an external influence on the model, representing genetic and obesity, we have considered the much discussed hypothesis $(65,71,74)$ that hyperinsulinemia can contribute to insulin resistance in addition to being a compensatory response to insulin resistance (Fig. 9). Any effect of increased insulin to induce insulin resistance has to be weighed against the effect

617 to enhance glucose uptake, and the outcome would depend on the net effect on the term $S_{I} I$ in Eq. 1. We

618 find that incorporating induced insulin resistance can either advance or delay the rate of progression to

619 T2D modestly depending on the strength of the two effects. We have not found support for the

620 hypothesis that it is the sole or primary initiating factor: to trigger T2D in the absence of intrinsic,

621 insulin-independent insulin resistance, we had to assume that the insulin-induced resistance is implausibly

622 strong. Similar results would apply if hyperinsulinemia resulted from increased secretion rather than

623 reduced clearance, but one would also have to consider whether in this case the increased beta-cell

624 workload might have an additional deleterious effect. 
626 We are aware of only one other modeling study (35) that has addressed the role of hyperinsulinemia as a

627 driver of diabetes pathogenesis. More specifically, that thoughtful analysis considered a variant of the

628 model of Topp et al (75) in which hypersecretion would reduce $S_{I}$. Under that assumption, and an

629 additional assumption that high insulin would impair beta-cell mass, hypersecretion was found to be able

630 to drive progression to T2D. However, increased insulin itself that has been shown to induce insulin

631 resistance (71), so we prefer to have insulin act directly on $S_{I}$. As we have said above, the effect of

632 hyperinsulinemia governed by that mechanism is modest.

633 The effects of induced insulin resistance are limited fundamentally because insulin both promotes glucose

634 uptake and contributes to resistance to that uptake. This is analogous to friction in mechanics. The

635 higher the insulin concentration, the more resistance is generated through increased negative feedback in

636 the insulin signaling cascade. Similarly, the faster a car goes, the more wind and rolling resistance it

637 generates. This resistance is not the motive force driving the car forward but a by-product of forward

638 motion that limits its speed. However, resistance is generally not strong enough to prevent acceleration, it

639 just requires more gas to achieve the desired speed. Similarly, the insulin resistance induced by insulin

640 can in most cases be overcome by secreting more insulin.

\section{Insulin secretion defects}

643 The simulations of progression to T2D (Figs. 1, 3, and 4) require some degree of secretion defect in

644 addition to the various combinations of insulin resistance. We assumed a defect only in the triggering

645 pathway $(\gamma)$, but we have obtained similar results by assuming defects in the amplifying pathway $(\sigma)$.

646 The assumed triggering defect represents a right shift in the glucose dose response curve, which 
647 correspond to a mild gain of function (GOF) mutation of the K(ATP) channels, a prominent GWAS hit

648 (55).

649 Assuming a more significant GOF mutation defect in the model leads to T2D with a lesser degree of

650 insulin resistance (Fig. S8). This is in line with the finding that K(ATP) defects are generally mild (for

651 example, the E23K mutation in the Kir6.2 component of K(ATP), KCNJ11, has an odds ratio of only 1.18

652 (33)), but they are fairly common and therefore may make significant contributions on the population

653 level in combination with insulin action defects.

654 If the K(ATP) defect is sufficiently severe, no insulin resistance is needed at all (Fig. S9). This is the 655 case in neonatal diabetes mellitus $(\mathrm{NDM})(4,47)$, which is of particular interest because it can be treated 656 effectively with sulfonylureas. This differs from typical T2D in adults, possibly because the drug brings

657 the genetically insufficiently active beta cells to a normal level of activity instead of overdriving them.

658 The examples of Figs. 2, 1, S8 and S9, in that order, lie on a continuum of progressively greater K(ATP) 659 channel function and lesser insulin resistance. As we showed previously in Fig. 5 of Ref. \#6, the greater 660 the degree of beta-cell dysfunction, the lesser the degree of insulin resistance required for T2D. In 661 addition to the degree of insulin resistance, the rate of decline of insulin sensitivity is important. Fig. S12 662 shows cases in which a slow decline is compensated whereas a fast decline is not, as shown previously in 663 (75). We emphasize that the requirement for insulin resistance for typical T2D progression is not in 664 contradiction with genome wide studies showing a predominance of beta-cell genes: most people who are 665 overweight or obese become insulin resistant, but only those with beta-cell defects become diabetic. Lean 666 individuals who are not insulin resistant can also develop diabetes, but only if they have severe and rare 667 secretion defects.

668 Although we have focused on the role of insulin resistance in promoting IFG, secretion defects can also 669 lead to IFG with little contribution of insulin resistance. Fig. S13 shows a case with a mild GOF mutation 
670 in K(ATP) channels, and Fig. S14 shows a case with a small readily releasable pool due to reduced

671 vesicle priming rate. With more extreme secretion defects, no insulin resistance is needed at all (not

672 shown). These patterns may be of particular relevance to Asian and possibly African populations where

673 secretion defects play a bigger role than insulin resistance in diabetes compared to those of European

674 descent.

675 Another secretory defect that may contribute to diabetes risk is impaired incretin signaling. Figure S15

676 illustrates that such an impairment would accelerate progression in a person predisposed to T2D.

677 The model shows that defects in first- and second-phase secretion are not independent. Impairment in one

678 leads to impairment in the other because of the harmful effects of elevated glucose. The model accounts

679 for classic data (13) showing that the acute insulin response to glucose (AIRg), a surrogate for first phase

680 secretion, declines with even modest increases in FPG during the NGT and pre-diabetic stages. The

681 model shows, however, that second-phase insulin secretion declines in parallel, belying a privileged role

682 for first-phase secretion, as also shown in another modeling study (36). In our model, reduced AIRg

683 results mainly from reduced size of the RRP, which can be caused by impairment in either first-phase or

684 second-phase secretion or both. More generally, any unanswered rise in either fasting or post-prandial

685 glucose impairs both first and second phase secretion, and any defect in either first or second phase

686 secretion raises both fasting and post-prandial glucose. This creates a vicious cycle that drives down both

687 first- and second-phase secretion regardless of which defect is primary. Empirical studies that do not

688 select for early isolated IFG or IGT cases do not show a clear prominence of first phase secretion loss

689 (31), in agreement with the model.

690 
692 We have also considered briefly glucose effectiveness, the ability of glucose to promote its own uptake

693 independent of insulin (Figs. S10, S11). For simplicity, we have assumed the differences in $E_{G 0}$ to be pre-

694 existing and constant in time. However, there is evidence that it is a regulated process; it has been

695 reported to be enhanced by FGF19 acting in the brain and may be related to the glucose intolerance

696 resulting from leptin deficiency in ob/ob mice (52). It may also account for the rescue by leptin of

697 glucose tolerance in insulin-deficient rodents treated with streptozotocin (32). Further progress in

698 modeling this process would benefit from identifying the molecular mechanism(s) underlying it, which

699 are currently not well understood.

\section{Limitations of the study}

703

704

705

706

707 they may be necessary to explain other pathways to T2D and will be addressed elsewhere.

712 To capture the full range of patterns, it is necessary to consider as well pre-existing variation in beta-cell

713 function, not the moment-to-moment beta-cell function, which evolves in response to hyperglycemia, but

The model as presented here is oversimplified in that it only considers glucotoxicity and neglects other factors that are likely to play a role, such as lipotoxicity (48). We are thus unable at present to address the possible role of hyperinsulinemia to promote hepatic fat accumulation (76). If prolonged, this could lead to liver damage and thus to a worsening of insulin resistance that could outlast the induced resistance we have modeled here (Eqs. 9 - 11), which would resolve if normal insulin levels were restored.

In addition, we believe that prolonged high secretion rate is probably harmful beyond the negative effect incorporated in the equation for $\sigma$, possibly because of ER stress and/or calcium toxicity (53). Those factors were not included here because they were not needed to account for the pathways considered, but the innate, genetic capacity of beta-cell function to adapt to hyperglycemia. For example, changing the 
715 parameters defining $\sigma_{\infty}$ (Eqs. 6, A16) has marked effects on the speed of progression in IGT; we

716 neglected this because it doesn't change the likelihood of entering the IGT state much.

717 We have modeled the suppression of hepatic glucose production as a direct effect of insulin on the liver.

718 However, much if not all of the acute effect of insulin is indirect, mediated by suppression of lipolysis in 719 adipose tissue, which reduces the supply of free fatty acid (FFA) to the liver (58). We consider this an 720 acceptable approximation for our purposes, as post-prandial suppression of lipolysis is roughly a mirror 721 image of the post-prandial rise in insulin. In future, if we want to account for FFA dynamics or adipose722 tissue insulin resistance, the model would have to be augmented.

723 We have modeled insulin-dependent glucose uptake as linear in insulin, but it is likely to be non-linear 724 (sigmoidal) (12) and has been suggested to exhibit hysteresis (78). We have not found these features to 725 be necessary to explain the data under consideration, but they can be easily added in the future if the need 726 arises.

727 We have neglected the role of excess glucagon secretion, which results from intrinsic defects in alpha 728 cells or from loss of paracrine regulation of alpha cells due to a relative lack of insulin $(21,56)$. This has 729 long been recognized to exacerbate post-prandial hyperglycemia in diabetes and pre-diabetes and is a 730 target of GLP-1-based insulin secretagogues. Without glucagon in the model, we are not able to simulate 731 fully the efficacy of that class of drugs, only their effect on insulin secretion. Hyperglucagonemia may 732 also contribute to hepatic insulin resistance, as proposed in recent reviews $(26,80)$ suggesting that 733 glucagon is part of a liver-alpha cell axis that is primarily devoted to regulating amino acid metabolism.

734 A primary form of glucagon resistance at the liver in this cycle could lead to hyperaminoacidemia and 735 hyperglucagonemia. The latter would contribute to excess hepatic glucose production and IFG in 736 addition to or in place of hepatic insulin resistance. This makes glucagon a tempting therapeutic target. 737 Hyperaminoacidemia and alpha-cell hyperplasia have thwarted many efforts, but some work in this direction continues (72). 
Other recent developments have shown that glucagon amplifies insulin secretion and thus under normal

740 conditions has a net hypoglycemic effect post-prandially $(16,82)$. Glucagon is thus now seen to be

741 homeostatic in both low and high glucose under normal conditions but can become anti-homeostatic in

742 diabetes or pre-diabetes. The emerging story is complex, and inclusion in the model is deferred to future

743 studies.

744 We have included only a very simple representation of insulin clearance, assuming a first-order

745 dependence on insulin concentration. We have not distinguished portal from peripheral insulin (63) or

746 considered possible regulation by glucose (60) or free fatty acids (7).

\section{Future directions}

749 This paper has demonstrated that if the insulin resistance phenotype of an individual is known, their

750 future trajectory of hyperglycemia can be predicted (Figs. 1 -4) and drug choice can potentially be

751 optimized for the patient's insulin resistance phenotype (Fig. 8). Another longitudinal model has shown

752 similarly the results of targeting insulin resistance vs. beta-cell replication (38). Our model can provide

753 similar predictions if the relative impairments in insulin secretion and insulin resistance are known. For

754 example, plotting $1 \mathrm{hPG}$ vs. 2hPG obtained by simulating OGTTs results in a family of trajectories similar

755 to the ones for FPG vs. 2hPG shown in Fig. 5. The differences in trajectories due to the contributions of

756 insulin secretion and insulin action can be read off from the difference between $1 \mathrm{hPG}$ and $2 \mathrm{hPG}$ and are

757 similarly most pronounced during prediabetes (Ha et al, ADA poster 1490-P, June, 2019). This

758 prediction may provide deeper insight into the diagnostic information that can be extracted from different

759 time points during the OGTT, an issue that is currently receiving much attention $(19,40)$.

760 Future versions of the model should include roles for glucagon, as described above. In addition, a more

761 complex model for insulin signaling would permit the investigation of the effects of particular defects in 
762 those pathways as well as possible mechanisms for the insulin resistance induced by insulin, which was

763 treated phenomenologically here.

764 In addition, to be a useful tool for patient stratification and treatment planning, one needs to ascertain the

765 patient's phenotype. We plan to investigate whether the model can be used, with suitable modifications,

766 to solve the inverse problem of inferring the individual's parameters of insulin resistance and beta-cell

767 function from the observed behavior.

\section{Acknowledgments}

770 The work was supported by the Intramural Research Program of the National Institutes of Health,

771 NIDDK. We thank Stephanie Chung, Richard Bertram, Over Cabrera, and Cecilia Diniz Behn for helpful

772 suggestions on the manuscript. 


\section{References}

775

776

777

778

779

780

781

782

783

784

785

786

787

788

789

790

791

792

793

794

795

796

797

798

799

800

801

802

803

804

805

806

807

808

1. Abdul-Ghani MA, Matsuda M, DeFronzo RA. Strong association between insulin resistance in liver and skeletal muscle in non-diabetic subjects. Diabet Med 25: 1289-1294, 2008. doi: 10.1111/j.1464-5491.2008.02597.x.

2. Ackerman E, Rosevear JW, McGuckin WF. A Mathematical Model of the Glucose-tolerance test. Phys Med Biol 9: 203-213, 1964. doi: 10.1088/0031-9155/9/2/307.

3. Ader M, Stefanovski D, Kim SP, Richey JM, lonut V, Catalano KJ, Hucking K, Ellmerer M, Van Citters G, Hsu IR, Chiu JD, Woolcott OO, Harrison LN, Zheng D, Lottati M, Kolka CM, Mooradian V, Dittmann J, Yae S, Liu H, Castro AVB, Kabir M, Bergman RN. Hepatic insulin clearance is the primary determinant of insulin sensitivity in the normal dog. Obesity (Silver Spring) 22: 1238-1245, 2014. doi: 10.1002/oby.20625.

4. Ashcroft FM, Puljung MC, Vedovato N. Neonatal Diabetes and the KATP Channel: From Mutation to Therapy. Trends Endocrinol Metab 28: 377-387, 2017. doi: 10.1016/j.tem.2017.02.003.

5. Bard Ermentrout. Simulating, Analyzing, and Animating Dynamical Systems [Online]. SIAM. http://www.math.pitt.edu/ bard/xpp/xpp.html.

6. Basu R, Barosa C, Jones J, Dube S, Carter R, Basu A, Rizza RA. Pathogenesis of prediabetes: role of the liver in isolated fasting hyperglycemia and combined fasting and postprandial hyperglycemia. $J$ Clin Endocrinol Metab 98: E409-417, 2013. doi: 10.1210/jc.2012-3056.

7. Bergman RN, Ader M. Free fatty acids and pathogenesis of type 2 diabetes mellitus. Trends Endocrinol Metab 11: 351-356, 2000.

8. Bergman RN, Ider YZ, Bowden CR, Cobelli C. Quantitative estimation of insulin sensitivity. Am J Physiol 236: E667-677, 1979.

9. Best JD, Kahn SE, Ader M, Watanabe RM, Ni TC, Bergman RN. Role of glucose effectiveness in the determination of glucose tolerance. Diabetes Care 19: 1018-1030, 1996. doi: 10.2337/diacare.19.9.1018.

10. Bodkin NL, Metzger BL, Hansen BC. Hepatic glucose production and insulin sensitivity preceding diabetes in monkeys. Am J Physiol 256: E676-681, 1989.

11. Bolie VW. Coefficients of normal blood glucose regulation. J Appl Physiol 16: 783-788, 1961. doi: 10.1152/jappl.1961.16.5.783.

12. Bonadonna RC, Groop L, Kraemer N, Ferrannini E, Del Prato S, DeFronzo RA. Obesity and insulin resistance in humans: a dose-response study. Metab Clin Exp 39: 452-459, 1990. doi:

10.1016/0026-0495(90)90002-t.

13. Brunzell JD, Robertson RP, Lerner RL, Hazzard WR, Ensinck JW, Bierman EL, Porte D. Relationships between fasting plasma glucose levels and insulin secretion during intravenous glucose tolerance tests. J Clin Endocrinol Metab 42: 222-229, 1976. doi: 10.1210/jcem-42-2-222. 
14. Brunzell JD, Robertson RP, Lerner RL, Hazzard WR, Ensinck JW, Bierman EL, Porte D. Relationships between fasting plasma glucose levels and insulin secretion during intravenous glucose tolerance tests. J Clin Endocrinol Metab 42: 222-229, 1976. doi: 10.1210/jcem-42-2-222.

15. Butler AE, Janson J, Bonner-Weir S, Ritzel R, Rizza RA, Butler PC. Beta-cell deficit and increased beta-cell apoptosis in humans with type 2 diabetes. Diabetes 52: 102-110, 2003. doi: 10.2337/diabetes.52.1.102.

16. Capozzi ME, Wait JB, Koech J, Gordon AN, Coch RW, Svendsen B, Finan B, D'Alessio DA, Campbell JE. Glucagon lowers glycemia when $\beta$-cells are active. JCI Insight 5, 2019. doi: 10.1172/jci.insight.129954.

17. Chen $\mathbf{Y}$, Wang S, Sherman A. Identifying the targets of the amplifying pathway for insulin secretion in pancreatic beta-cells by kinetic modeling of granule exocytosis. Biophys J 95: 2226-2241, 2008. doi: 10.1529/biophysj.107.124990.

18. Choy S, Kjellsson MC, Karlsson MO, de Winter W. Weight-HbA1c-insulin-glucose model for describing disease progression of type 2 diabetes. CPT Pharmacometrics Syst Pharmacol 5: 11-19, 2016. doi: $10.1002 / p s p 4.12051$.

19. Chung ST, Ha J, Onuzuruike AU, Kasturi K, Galvan-De La Cruz M, Bingham BA, Baker RL, Utumatwishima JN, Mabundo LS, Ricks M, Sherman AS, Sumner AE. Time to glucose peak during an oral glucose tolerance test identifies prediabetes risk. .

20. Cobelli C, Dalla Man C, Toffolo G, Basu R, Vella A, Rizza R. The oral minimal model method. Diabetes 63: 1203-1213, 2014. doi: 10.2337/db13-1198.

21. D'Alessio D. The role of dysregulated glucagon secretion in type 2 diabetes. Diabetes Obes Metab 13 Suppl 1: 126-132, 2011. doi: 10.1111/j.1463-1326.2011.01449.x.

22. Dankner R, Chetrit A, Shanik MH, Raz I, Roth J. Basal-state hyperinsulinemia in healthy normoglycemic adults is predictive of type 2 diabetes over a 24-year follow-up: a preliminary report. Diabetes Care 32: 1464-1466, 2009. doi: 10.2337/dc09-0153.

23. De Gaetano A, Hardy T, Beck B, Abu-Raddad E, Palumbo P, Bue-Valleskey J, Pørksen N. Mathematical models of diabetes progression. Am J Physiol Endocrinol Metab 295: E1462-1479, 2008. doi: 10.1152/ajpendo.90444.2008.

24. De Gaetano A, Hardy TA. A novel fast-slow model of diabetes progression: Insights into mechanisms of response to the interventions in the Diabetes Prevention Program. PLOS ONE 14: e0222833, 2019. doi: 10.1371/journal.pone.0222833.

25. de Winter W, DeJongh J, Post T, Ploeger B, Urquhart R, Moules I, Eckland D, Danhof M. A mechanism-based disease progression model for comparison of long-term effects of pioglitazone, metformin and gliclazide on disease processes underlying Type 2 Diabetes Mellitus. J Pharmacokinet Pharmacodyn 33: 313-343, 2006. doi: 10.1007/s10928-006-9008-2. 
26. Dean ED. A Primary Role for $\alpha$-Cells as Amino Acid Sensors. Diabetes 69: 542-549, 2020. doi: 10.2337/dbi19-0021.

27. DeFronzo RA. Lilly lecture 1987. The triumvirate: beta-cell, muscle, liver. A collusion responsible for NIDDM. Diabetes 37: 667-687, 1988.

28. DeFronzo RA, Tripathy D. Skeletal Muscle Insulin Resistance Is the Primary Defect in Type 2 Diabetes. Diabetes Care 32: S157-S163, 2009. doi: 10.2337/dc09-S302.

29. DREAM (Diabetes REduction Assessment with ramipril and rosiglitazone Medication) Trial Investigators, Gerstein HC, Yusuf S, Bosch J, Pogue J, Sheridan P, Dinccag N, Hanefeld M, Hoogwerf B, Laakso M, Mohan V, Shaw J, Zinman B, Holman RR. Effect of rosiglitazone on the frequency of diabetes in patients with impaired glucose tolerance or impaired fasting glucose: a randomised controlled trial. Lancet 368: 1096-1105, 2006. doi: 10.1016/S0140-6736(06)69420-8.

30. Dube S, Errazuriz-Cruzat I, Basu A, Basu R. The forgotten role of glucose effectiveness in the regulation of glucose tolerance. Curr Diab Rep 15: 605, 2015. doi: 10.1007/s11892-015-0605-6.

31. Gerich JE. Is reduced first-phase insulin release the earliest detectable abnormality in individuals destined to develop type 2 diabetes? Diabetes 51 Suppl 1: S117-121, 2002.

32. German JP, Thaler JP, Wisse BE, Oh-I S, Sarruf DA, Matsen ME, Fischer JD, Taborsky GJ, Schwartz MW, Morton GJ. Leptin activates a novel CNS mechanism for insulin-independent normalization of severe diabetic hyperglycemia. Endocrinology 152: 394-404, 2011. doi: 10.1210/en.2010-0890.

33. Gloyn AL, Weedon MN, Owen KR, Turner MJ, Knight BA, Hitman G, Walker M, Levy JC, Sampson M, Halford S, McCarthy MI, Hattersley AT, Frayling TM. Large-scale association studies of variants in genes encoding the pancreatic beta-cell KATP channel subunits Kir6.2 (KCNJ11) and SUR1 (ABCC8) confirm that the KCNJ11 E23K variant is associated with type 2 diabetes. Diabetes 52: 568-572, 2003. doi: 10.2337/diabetes.52.2.568.

34. Glynn E, Thompson B, Vadrevu S, Lu S, Kennedy RT, Ha J, Sherman A, Satin LS. Chronic Glucose Exposure Systematically Shifts the Oscillatory Threshold of Mouse Islets: Experimental Evidence for an Early Intrinsic Mechanism of Compensation for Hyperglycemia. Endocrinology 157: 611-623, 2016. doi: 10.1210/en.2015-1563.

35. Goel P. Insulin resistance or hypersecretion? The ßIG picture revisited. J Theor Biol 384: 131-139, 2015. doi: 10.1016/j.jtbi.2015.07.033.

36. Grespan E, Giorgino T, Arslanian S, Natali A, Ferrannini E, Mari A. Defective Amplifying Pathway of $\beta$-cell Secretory Response to Glucose in Type 2 Diabetes: Integrated Modeling of in vitro and in vivo Evidence. .

37. Ha J, Satin LS, Sherman AS. A Mathematical Model of the Pathogenesis, Prevention, and Reversal of Type 2 Diabetes. Endocrinology 157: 624-635, 2016. doi: 10.1210/en.2015-1564. 
38. Hardy T, Abu-Raddad E, Porksen N, De Gaetano A. Evaluation of a mathematical model of diabetes progression against observations in the Diabetes Prevention Program. Am J Physiol Endocrinol Metab 303: E200-212, 2012. doi: 10.1152/ajpendo.00421.2011.

39. Henquin JC. Triggering and amplifying pathways of regulation of insulin secretion by glucose. Diabetes 49: 1751-1760, 2000.

40. Hulman A, Witte DR, Vistisen D, Balkau B, Dekker JM, Herder C, Hatunic M, Konrad T, Færch K, Manco M. Pathophysiological Characteristics Underlying Different Glucose Response Curves: A Latent Class Trajectory Analysis From the Prospective EGIR-RISC Study. Diabetes Care 41: 17401748, 2018. doi: 10.2337/dc18-0279.

41. Jones CN, Pei D, Staris P, Polonsky KS, Chen YD, Reaven GM. Alterations in the glucose-stimulated insulin secretory dose-response curve and in insulin clearance in nondiabetic insulin-resistant individuals. J Clin Endocrinol Metab 82: 1834-1838, 1997. doi: 10.1210/jcem.82.6.3979.

42. Jung S-H, Jung $\mathbf{C}-\mathbf{H}$, Reaven GM, Kim SH. Adapting to insulin resistance in obesity: role of insulin secretion and clearance. Diabetologia 61: 681-687, 2018. doi: 10.1007/s00125-017-4511-0.

43. Karin O, Swisa A, Glaser B, Dor Y, Alon U. Dynamical compensation in physiological circuits. Mol Syst Biol 12: 886, 2016.

44. Kim MK, Reaven GM, Kim SH. Dissecting the relationship between obesity and hyperinsulinemia: Role of insulin secretion and insulin clearance. Obesity (Silver Spring) 25: 378-383, 2017. doi: 10.1002/oby.21699.

45. Knowler WC, Barrett-Connor E, Fowler SE, Hamman RF, Lachin JM, Walker EA, Nathan DM, Diabetes Prevention Program Research Group. Reduction in the incidence of type 2 diabetes with lifestyle intervention or metformin. N Engl J Med 346: 393-403, 2002. doi: 10.1056/NEJMoa012512.

46. Kodama K, Tojjar D, Yamada S, Toda K, Patel CJ, Butte AJ. Ethnic differences in the relationship between insulin sensitivity and insulin response: a systematic review and meta-analysis. Diabetes Care 36: 1789-1796, 2013. doi: 10.2337/dc12-1235.

47. Koster JC, Remedi MS, Dao C, Nichols CG. ATP and sulfonylurea sensitivity of mutant ATP-sensitive $\mathrm{K}+$ channels in neonatal diabetes: implications for pharmacogenomic therapy. Diabetes 54: 26452654, 2005. doi: 10.2337/diabetes.54.9.2645.

48. Lim EL, Hollingsworth KG, Aribisala BS, Chen MJ, Mathers JC, Taylor R. Reversal of type 2 diabetes: normalisation of beta cell function in association with decreased pancreas and liver triacylglycerol. Diabetologia 54: 2506-2514, 2011. doi: 10.1007/s00125-011-2204-7.

49. Mari A, Ferrannini E. Beta-cell function assessment from modelling of oral tests: an effective approach. Diabetes Obes Metab 10 Suppl 4: 77-87, 2008. doi: 10.1111/j.1463-1326.2008.00946.x.

50. Mason CC, Hanson RL, Knowler WC. Progression to type 2 diabetes characterized by moderate then rapid glucose increases. Diabetes 56: 2054-2061, 2007. doi: 10.2337/db07-0053. 
51. Meigs JB, Muller DC, Nathan DM, Blake DR, Andres R, Baltimore Longitudinal Study of Aging. The natural history of progression from normal glucose tolerance to type 2 diabetes in the Baltimore Longitudinal Study of Aging. Diabetes 52: 1475-1484, 2003.

52. Morton GJ, Matsen ME, Bracy DP, Meek TH, Nguyen HT, Stefanovski D, Bergman RN, Wasserman DH, Schwartz MW. FGF19 action in the brain induces insulin-independent glucose lowering. J Clin Invest 123: 4799-4808, 2013. doi: 10.1172/JCI70710.

53. Nichols CG, Remedi MS. The diabetic $\beta$-cell: hyperstimulated vs. hyperexcited. Diabetes Obes Metab 14: 129-135, 2012. doi: 10.1111/j.1463-1326.2012.01655.x.

54. Nyman E, Rozendaal YJW, Helmlinger G, Hamrén B, Kjellsson MC, Strålfors P, van Riel NAW, Gennemark P, Cedersund G. Requirements for multi-level systems pharmacology models to reach end-usage: the case of type 2 diabetes. Interface Focus 6: 20150075, 2016. doi: 10.1098/rsfs.2015.0075.

55. Odgerel Z, Lee HS, Erdenebileg N, Gandbold S, Luvsanjamba M, Sambuughin N, Sonomtseren S, Sharavdorj P, Jodov E, Altaisaikhan K, Goldfarb LG. Genetic variants in potassium channels are associated with type 2 diabetes in a Mongolian population. J Diabetes 4: 238-242, 2012. doi: 10.1111/j.1753-0407.2011.00177.x.

56. Omar-Hmeadi M, Lund P-E, Gandasi NR, Tengholm A, Barg S. Paracrine control of $\alpha$-cell glucagon exocytosis is compromised in human type-2 diabetes. Nat Commun 11: 1896, 2020. doi: 10.1038/s41467-020-15717-8.

57. Perreault L, Bergman BC, Playdon MC, Dalla Man C, Cobelli C, Eckel RH. Impaired fasting glucose with or without impaired glucose tolerance: progressive or parallel states of prediabetes? Am J Physiol Endocrinol Metab 295: E428-435, 2008. doi: 10.1152/ajpendo.90354.2008.

58. Perry RJ, Camporez J-PG, Kursawe R, Titchenell PM, Zhang D, Perry CJ, Jurczak MJ, Abudukadier A, Han MS, Zhang X-M, Ruan H-B, Yang X, Caprio S, Kaech SM, Sul HS, Birnbaum MJ, Davis RJ, Cline GW, Petersen KF, Shulman GI. Hepatic acetyl CoA links adipose tissue inflammation to hepatic insulin resistance and type 2 diabetes. Cell 160: 745-758, 2015. doi: 10.1016/j.cell.2015.01.012.

59. Petersen MC, Shulman GI. Mechanisms of Insulin Action and Insulin Resistance. Physiol Rev 98: 2133-2223, 2018. doi: 10.1152/physrev.00063.2017.

60. Piccinini F, Dalla Man C, Vella A, Cobelli C. A Model for the Estimation of Hepatic Insulin Extraction After a Meal. IEEE Transactions on Biomedical Engineering 63: 1925-1932, 2016. doi: 10.1109/TBME.2015.2505507.

61. Piccinini F, Polidori DC, Gower BA, Bergman RN. Hepatic but Not Extrahepatic Insulin Clearance Is Lower in African American Than in European American Women. Diabetes 66: 2564-2570, 2017. doi: $10.2337 / \mathrm{db} 17-0413$.

62. Piller C. Dubious diagnosis. Science 363: 1026-1031, 2019. doi: 10.1126/science.363.6431.1026. 
63. Polidori D, Bergman RN, Sumner AE. A New Model-Based Method for Estimating Hepatic and Peripheral Insulin Clearance from Intravenous Glucose Tolerance Test Data (2015 ADA abstract 254-LB). [date unknown].

64. Porat S, Weinberg-Corem N, Tornovsky-Babaey S, Schyr-Ben-Haroush R, Hija A, Stolovich-Rain M, Dadon D, Granot Z, Ben-Hur V, White P, Girard CA, Karni R, Kaestner KH, Ashcroft FM, Magnuson MA, Saada A, Grimsby J, Glaser B, Dor Y. Control of pancreatic $\beta$ cell regeneration by glucose metabolism. Cell Metab 13: 440-449, 2011. doi: 10.1016/j.cmet.2011.02.012.

65. Pories WJ, Dohm GL. Diabetes: have we got it all wrong? Hyperinsulinism as the culprit: surgery provides the evidence. Diabetes Care 35: 2438-2442, 2012. doi: 10.2337/dc12-0684.

66. Rahier J, Guiot Y, Goebbels RM, Sempoux C, Henquin JC. Pancreatic beta-cell mass in European subjects with type 2 diabetes. Diabetes Obes Metab 10 Suppl 4: 32-42, 2008. doi: 10.1111/j.14631326.2008.00969.x.

67. Ribbing J, Hamrén B, Svensson MK, Karlsson MO. A model for glucose, insulin, and beta-cell dynamics in subjects with insulin resistance and patients with type 2 diabetes. $J$ Clin Pharmacol 50: 861-872, 2010. doi: 10.1177/0091270009349711.

68. RISE Consortium. Lack of Durable Improvements in $\beta$-Cell Function Following Withdrawal of Pharmacological Interventions in Adults With Impaired Glucose Tolerance or Recently Diagnosed Type 2 Diabetes. Diabetes Care 42: 1742-1751, 2019. doi: 10.2337/dc19-0556.

69. Saisho Y, Butler AE, Manesso E, Elashoff D, Rizza RA, Butler PC. $\beta$-cell mass and turnover in humans: effects of obesity and aging. Diabetes Care 36: 111-117, 2013. doi: 10.2337/dc12-0421.

70. Sedaghat AR, Sherman A, Quon MJ. A mathematical model of metabolic insulin signaling pathways. Am J Physiol Endocrinol Metab 283: E1084-1101, 2002. doi: 10.1152/ajpendo.00571.2001.

71. Shanik MH, Xu Y, Skrha J, Dankner R, Zick Y, Roth J. Insulin resistance and hyperinsulinemia: is hyperinsulinemia the cart or the horse? Diabetes Care 31 Suppl 2: S262-268, 2008. doi: $10.2337 / \mathrm{dc08}-\mathrm{s} 264$.

72. Sharma AX, Quittner-Strom EB, Lee Y, Johnson JA, Martin SA, Yu X, Li J, Lu J, Cai Z, Chen S, Wang M-Y, Zhang Y, Pearson MJ, Dorn AC, McDonald JG, Gordillo R, Yan H, Thai D, Wang ZV, Unger RH, Holland WL. Glucagon Receptor Antagonism Improves Glucose Metabolism and Cardiac Function by Promoting AMP-Mediated Protein Kinase in Diabetic Mice. Cell Rep 22: 1760-1773, 2018. doi: 10.1016/j.celrep.2018.01.065.

73. Tabák AG, Jokela M, Akbaraly TN, Brunner EJ, Kivimäki M, Witte DR. Trajectories of glycaemia, insulin sensitivity, and insulin secretion before diagnosis of type 2 diabetes: an analysis from the Whitehall II study. Lancet 373: 2215-2221, 2009. doi: 10.1016/S0140-6736(09)60619-X.

74. Thomas DD, Corkey BE, Istfan NW, Apovian CM. Hyperinsulinemia: An Early Indicator of Metabolic Dysfunction. J Endocr Soc 3: 1727-1747, 2019. doi: 10.1210/js.2019-00065. 
75. Topp B, Promislow K, deVries G, Miura RM, Finegood DT. A model of beta-cell mass, insulin, and glucose kinetics: pathways to diabetes. J Theor Biol 206: 605-619, 2000. doi: 10.1006/jtbi.2000.2150.

76. Tricò D, Galderisi A, Mari A, Polidori D, Galuppo B, Pierpont B, Samuels S, Santoro N, Caprio S. Intrahepatic fat, irrespective of ethnicity, is associated with reduced endogenous insulin clearance and hepatic insulin resistance in obese youths: a cross-sectional and longitudinal study from the Yale Pediatric NAFLD cohort. .

77. Utumatwishima JN, Chung ST, Bentley AR, Udahogora M, Sumner AE. Reversing the tide - diagnosis and prevention of T2DM in populations of African descent. Nat Rev Endocrinol 14: 45-56, 2018. doi: 10.1038/nrendo.2017.127.

78. Wang G. Raison d'être of insulin resistance: the adjustable threshold hypothesis. $J R$ Soc Interface 11: 20140892, 2014. doi: 10.1098/rsif.2014.0892.

79. Wang Y-F, Khan M, van den Berg HA. Interaction of fast and slow dynamics in endocrine control systems with an application to $\beta$-cell dynamics. Math Biosci 235: 8-18, 2012. doi: 10.1016/j.mbs.2011.10.003.

80. Wewer Albrechtsen NJ, Pedersen J, Galsgaard KD, Winther-Sørensen M, Suppli MP, Janah L, Gromada J, Vilstrup H, Knop FK, Holst JJ. The Liver- $\alpha$-Cell Axis and Type 2 Diabetes. Endocr Rev 40: 1353-1366, 2019. doi: 10.1210/er.2018-00251.

81. Yki-Järvinen H. Thiazolidinediones and the liver in humans. Curr Opin Lipidol 20: 477-483, 2009. doi: 10.1097/MOL.0b013e3283321d37.

82. Zhu L, Dattaroy D, Pham J, Wang L, Barella LF, Cui Y, Wilkins KJ, Roth BL, Hochgeschwender U, Matschinsky FM, Kaestner KH, Doliba NM, Wess J. Intra-islet glucagon signaling is critical for maintaining glucose homeostasis. JCI Insight 5, 2019. doi: 10.1172/jci.insight.127994. 
1009

1010

1011

1012

1013

1014

1015

1016

1017

1018

1019

1020

1021

1022

1023

1024

1025

1026

1027

1028

1029

1030

1031

1032

1033

1034

1035

1036

1037

1038

1039

1040

1041

1042

1043

1044

1045

1046

Figure 1: IGT-first pathway to diabetes. (A) Assumed severe decline in peripheral insulin sensitivity. (B) Assumed mild decline in hepatic insulin sensitivity. (C) Simulated longitudinal changes based on the assumptions in (A) and (B) in fasting plasma glucose (FPG) and two-hour glucose (2hPG) during OGTTs performed at each time point. The virtual subject experiences first high two-hour glucose (IGT), then high fasting glucose (CGI), and finally crosses the $2 \mathrm{hPG}$ threshold for T2D. (D) Simulated longitudinal changes in fasting plasma insulin (FPI) and two-hour insulin (2hPI) during the OGTTs. Insulin increases early on but decreases later. (E) The component of $\beta$-cell function represented by $\sigma$ decreases progressively throughout. (F) The $\beta$-cell mass, $\beta$, first increases during prediabetes, then decreases after diabetes onset.

Figure 2: Insulin resistance (same as Fig. 1) does not lead to diabetes if beta-cell function is sufficiently responsive. The $\gamma$-dynamics is made stronger than in Fig. 1 by decreasing $\gamma_{s}$ to $90 \mathrm{mg} / \mathrm{dl}$ from $100 \mathrm{mg} / \mathrm{dl}$ (see other parameters in Table S12A). (A) Assumed severe decline in peripheral insulin sensitivity. (B) Assumed mild decline in hepatic insulin sensitivity. (C) Simulated longitudinal changes in FPG and 2hPG during OGTTs performed at each time point. The virtual subject experiences modest rises in glucose and crosses the threshold for IGT but never crosses the thresholds for IFG, CGI or T2D. (D) Simulated longitudinal changes in fasting plasma insulin (FPI) and two-hour insulin (2hPI) during the OGTTs. Insulin concentration increases and saturates but never declines. (E) The $\beta$-cell function component $\sigma$ first decreases, but then levels off. Increase in the $\beta$-cell function component represented by $\gamma$ (not shown) helps limit the rise in glucose, which allows the $\beta$-cell mass $\beta$ to increase gradually throughout $(F)$.

Figure 3: IFG-first pathway to diabetes. (A) Assumed mild decline in peripheral insulin sensitivity. (B) Assumed severe decline in hepatic insulin sensitivity component hepa $a_{S I}$. (C) Simulated longitudinal changes in FPG and 2hPG during OGTTs performed at each time point. The virtual subject experiences first high FPG (IFG), then high 2hPG (CGI), and finally crosses the FPG threshold for T2D first. Simulated longitudinal changes in fasting plasma insulin (FPI) and two-hour insulin (2hPI) during the OGTTs. Insulin concentration increases early on but decreases later. (E) The $\beta$-cell function component $\sigma$ decreases progressively throughout. (F) The $\beta$-cell mass $\beta$ increases during prediabetes then decreases after diabetes onset.

Figure 4: The CGI state is not obligatory. (A), (B): Extreme discrepancy between peripheral and hepatic $(\mathrm{A}-\mathrm{B})$ insulin resistance results in progression directly from IGT to T2D without passing through CGI based on OGTTs performed at each time point (C). FPG does not exceed the threshold for IFG until after T2D onset. (D) Simulated longitudinal changes in fasting plasma insulin (FPI) and two-hour insulin (2hPI) during the OGTTs. Insulin increases early on, then decreases. (E) The $\beta$-cell function component $\sigma$ decreases more rapidly than in Figs. 1, 3. (F) The $\beta$-cell mass $\beta$ increases during prediabetes, then decreases after diabetes onset.

Figure 5: (A) Two-hour glucose (2hPG) plotted vs. fasting glucose (FPG) for varying degrees of peripheral insulin sensitivity, $S_{I}$, and hepatic insulin sensitivity hepa $a_{S I}$, starting from the same initial values as in Figs. $1-4$ and evolving to the target values, $\operatorname{tar}_{S I}$ (Eq. A13) and tarhepasI (Eq. A14) as follows: from upper left to lower right, the $\operatorname{tar}_{S I}$ values increase: $0.1,0.17,0.22,0.33,0.4$ and 0.5 ; and the 
1047

1048

1049

1050

1051

1052

1053

1054

1055

1056

1057

1058

1059

1060

1061

1062

1063

1064

1065

1066

1067

1068

1069

1070

1071

1072

1073

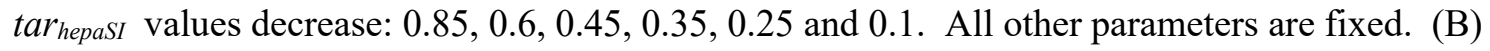
expanded view of (A) to highlight the prediabetes region.

Figure 6: Glucose during OGTTs performed at the times indicated by the black dots in (A) Fig. 1 (IGTfirst pathway) and (B) Fig. 3 (IFG-first pathway). (C, D) insulin corresponding to A and B, respectively. See text for details.

Figure 7: Insulin during IVGTTs performed at the time points indicated by the black dots in (A) Fig. 1 (IGT-first pathway) and (B) Fig. 3 (IFG-first pathway). (C, D): RRP size (numbers of vesicles) corresponding to A and B, respectively. Decline of AIRg parallels the reduction of RRP for each case.

Figure 8: Drug therapies targeting either peripheral insulin sensitivity (modeled as a rapid increase in $S_{I}$ ) or hepatic insulin sensitivity (modeled as a rapid increase in hepa $a_{S i}$; see Tables S13 - S16 for details). The drugs are applied in the early stages of T2D (A, B) or during prediabetes $(C, D)$. (A, C) Dominant peripheral insulin resistance, leading to IGT-first pathway as in Fig. 1. (B, D) Dominant hepatic insulin resistance, leading to IFG-first pathway as in Fig. 3. The appropriately targeted drug is more effective than the inappropriately targeted drug in each case, but therapy is more effective when initiated during prediabetes.

Figure 9: Effects of insulin clearance and insulin-induced insulin resistance. The case of Fig. 1 (IGT first) is re-simulated with varying rates of insulin clearance and induced insulin resistance, defined by Eqs. 13 16. Panel A: $S_{I}=S_{I, 0} E$, where $S_{I, 0}$ is calculated as in Fig. 1 and $E$ is the induced effect plotted in Panel F. Legend: black (control), same as Fig. 1, but with insulin clearance $k=0.4861 \mathrm{~min}^{-1}$, essentially no induced effect $\left(\alpha_{E}=300 \mu \mathrm{U} / \mathrm{ml}\right) ;$ green: reduced clearance $\left(k=0.37499 \mathrm{~min}^{-1}\right)$, modest induced effect $\left(\alpha_{E}=50 \mu \mathrm{U} / \mathrm{ml}\right)$; blue: $k=0.37499 \mathrm{~min}^{-1}$, stronger induced effect $\left(\alpha_{E}=15 \mu \mathrm{U} / \mathrm{ml}\right)$; red: $k=0.37499$ $\min ^{-1}$, very strong induced effect $\left(\alpha_{E}=5 \mu \mathrm{U} / \mathrm{ml}\right)$. Other parameters are listed in Table S17. 
Fig. 1
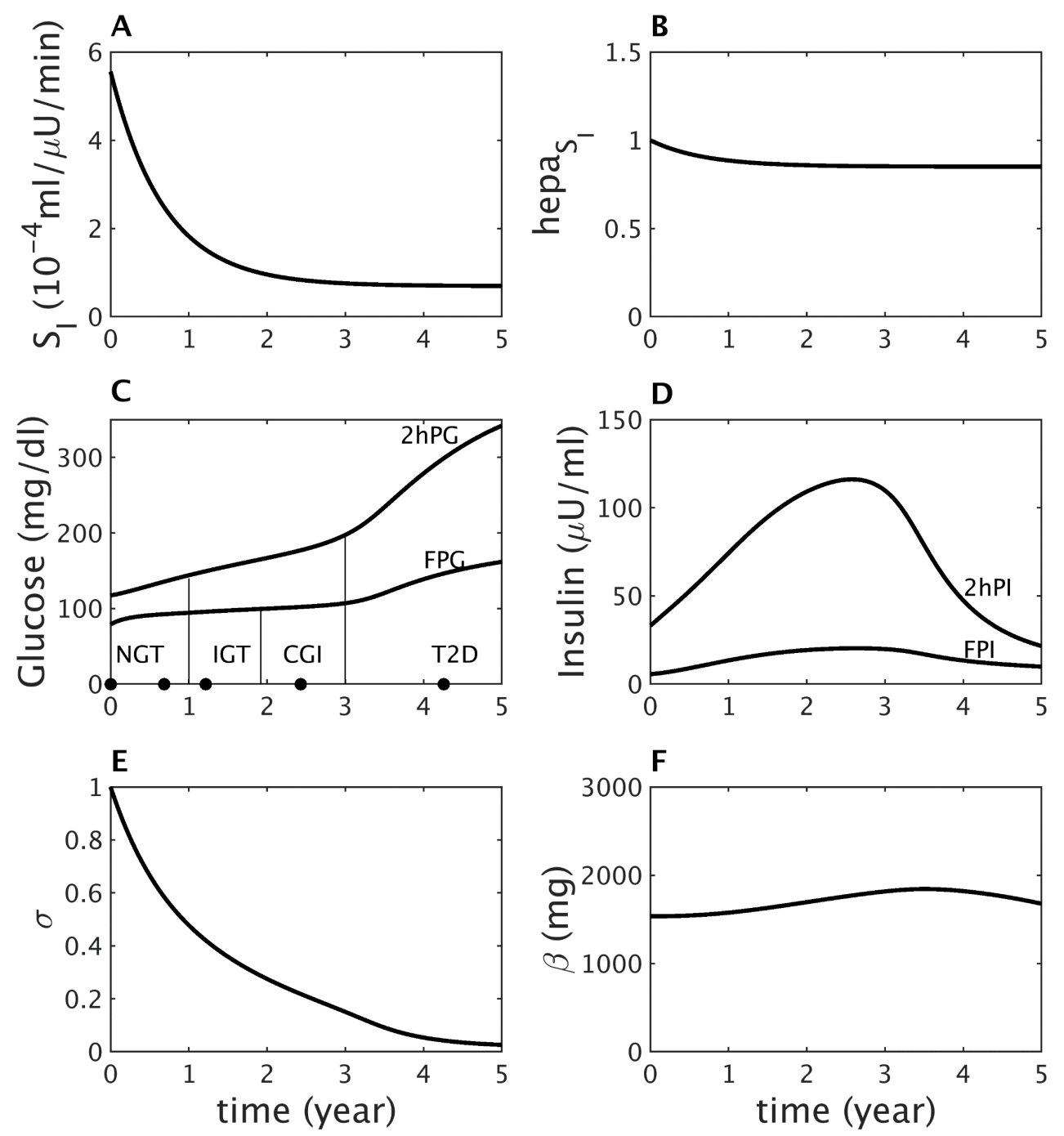
Fig. 2
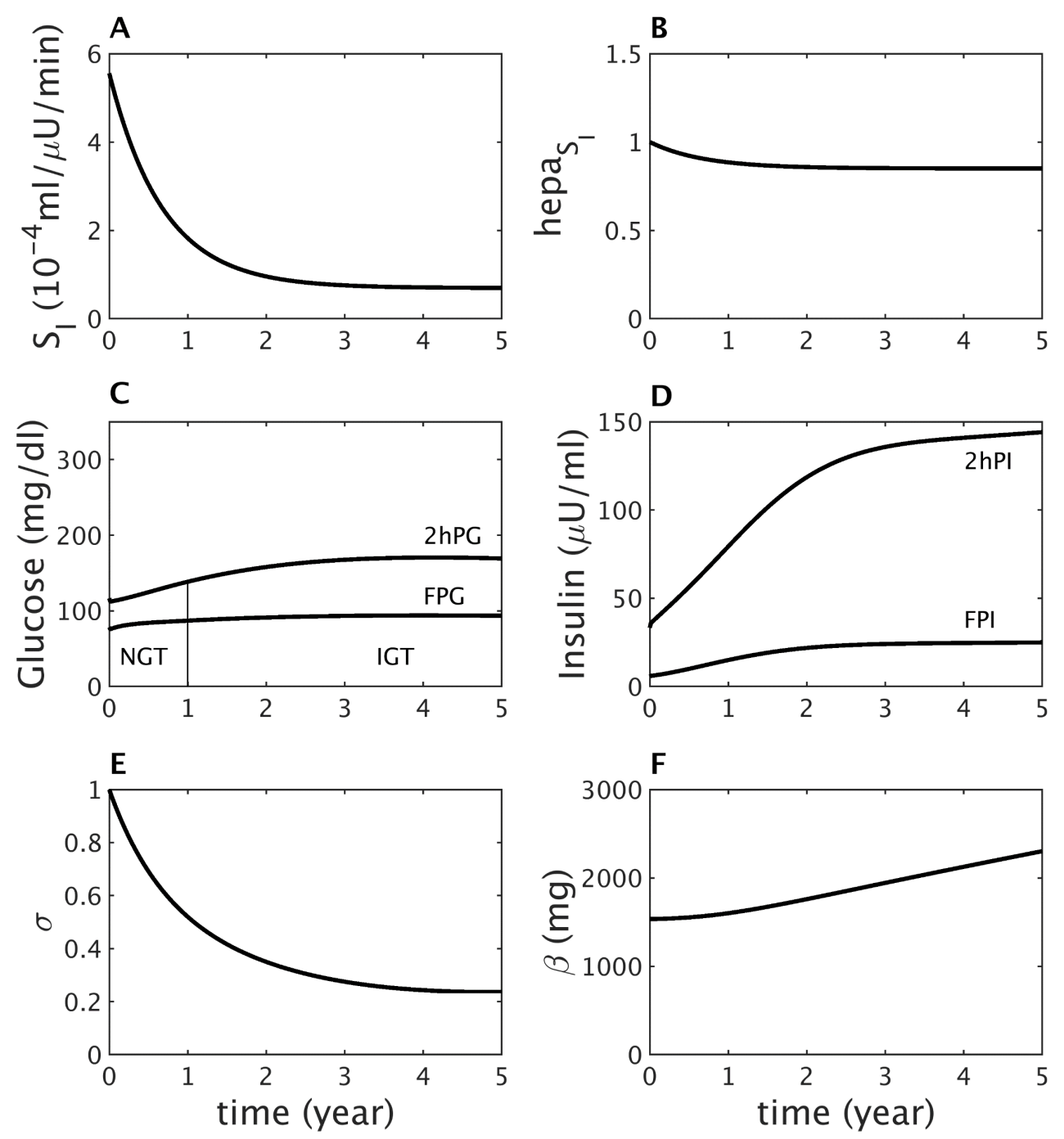
Fig. 3
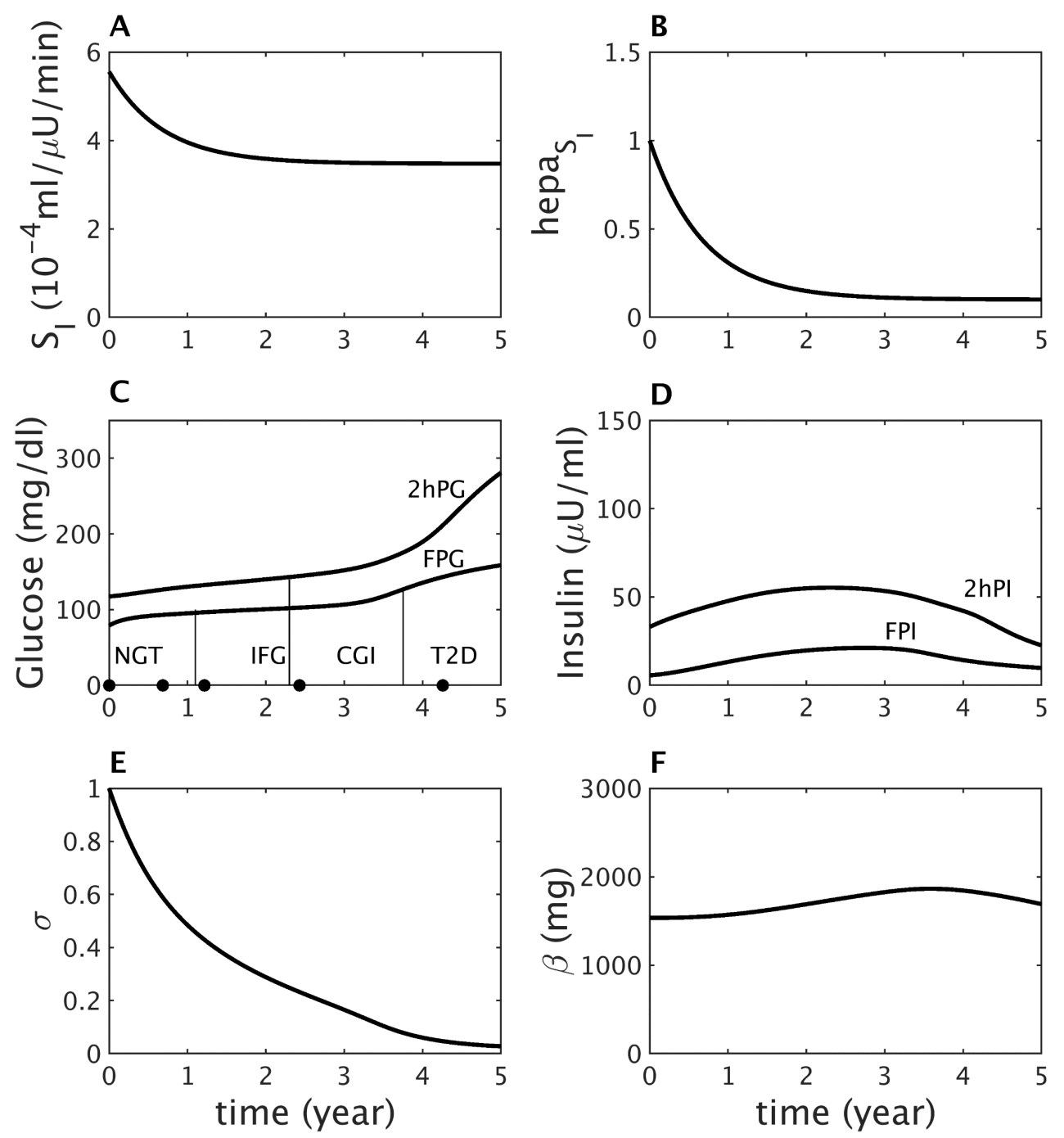
Fig. 4
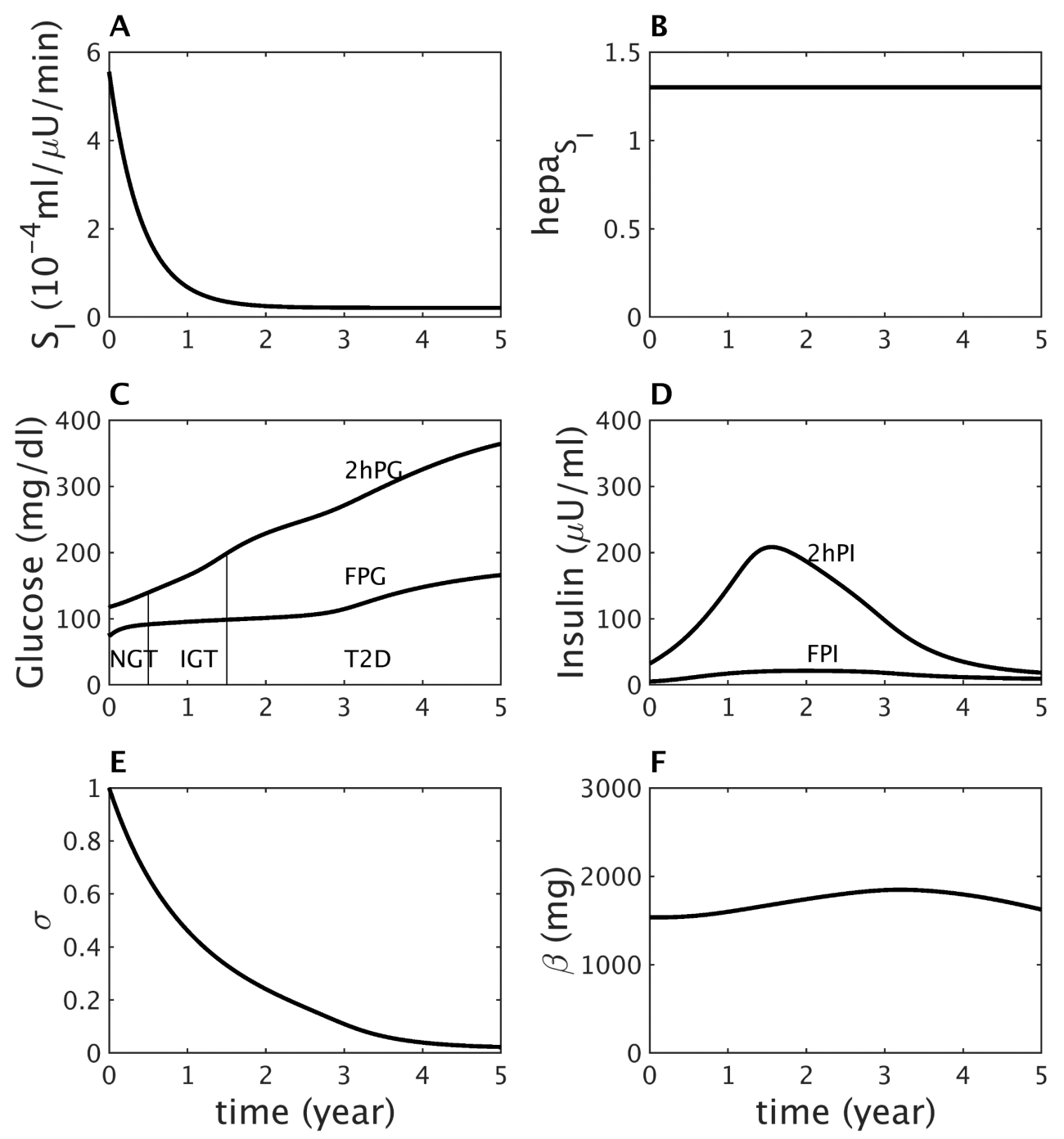
Fig. 5

A

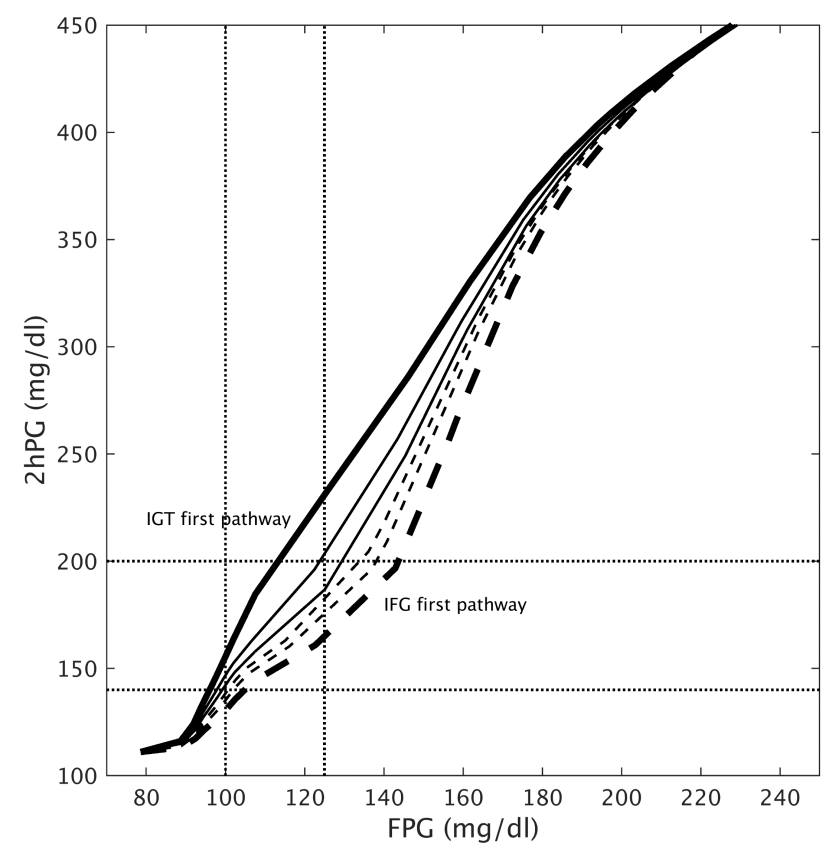

B

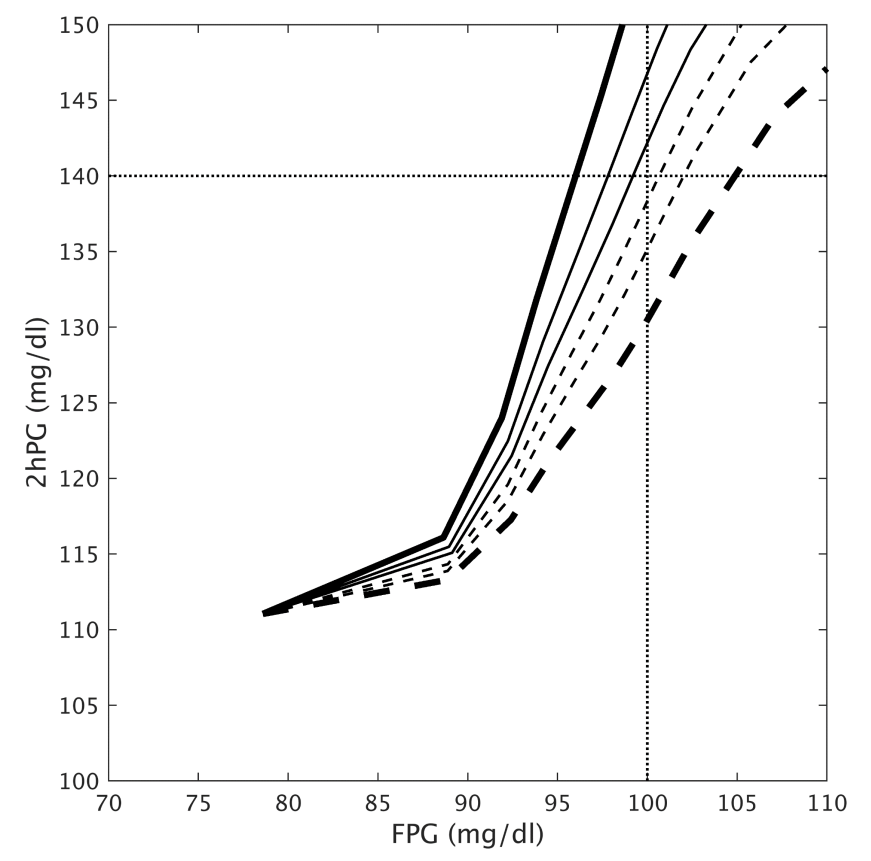




\section{Fig. 6}
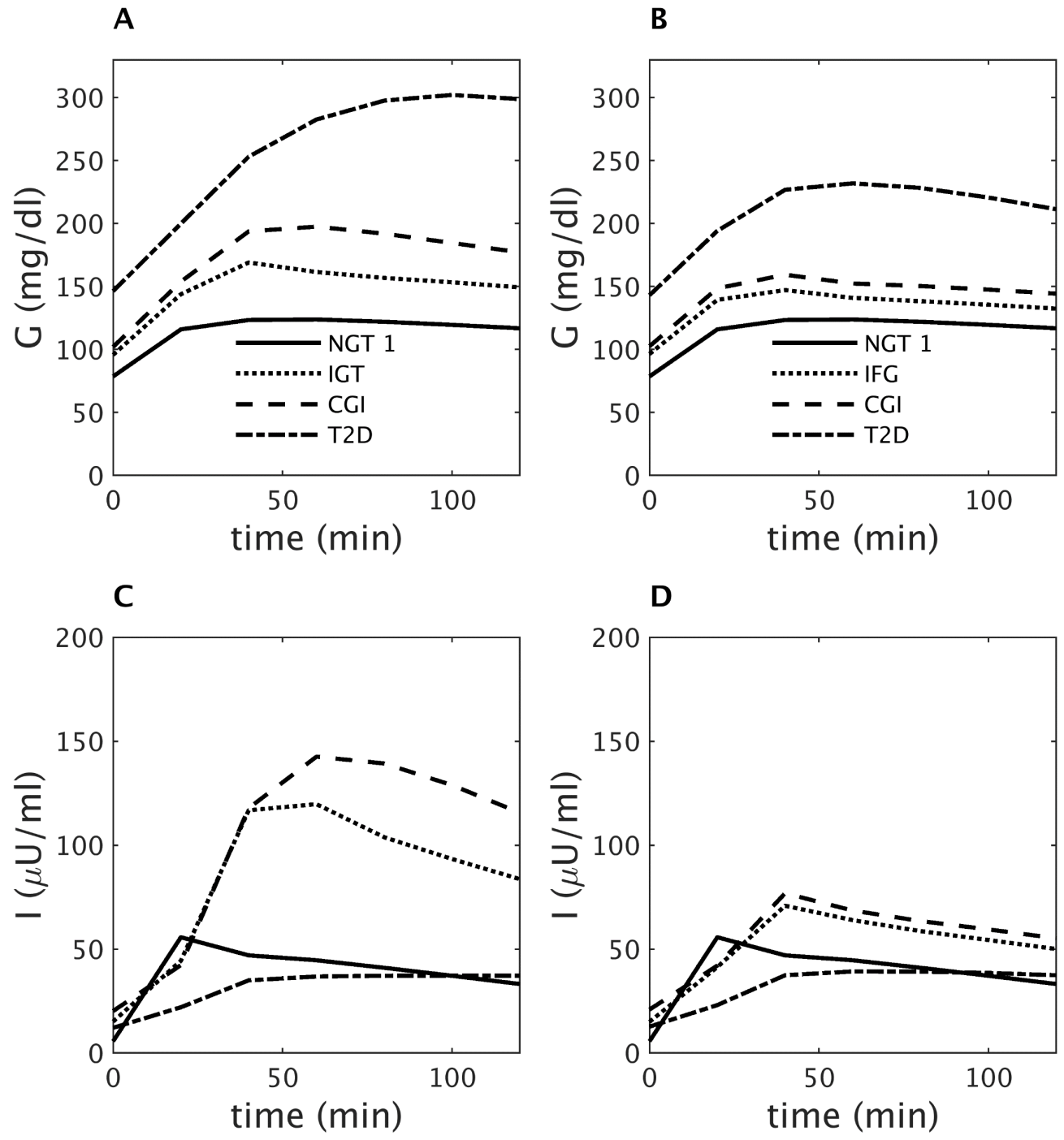
Fig. 7
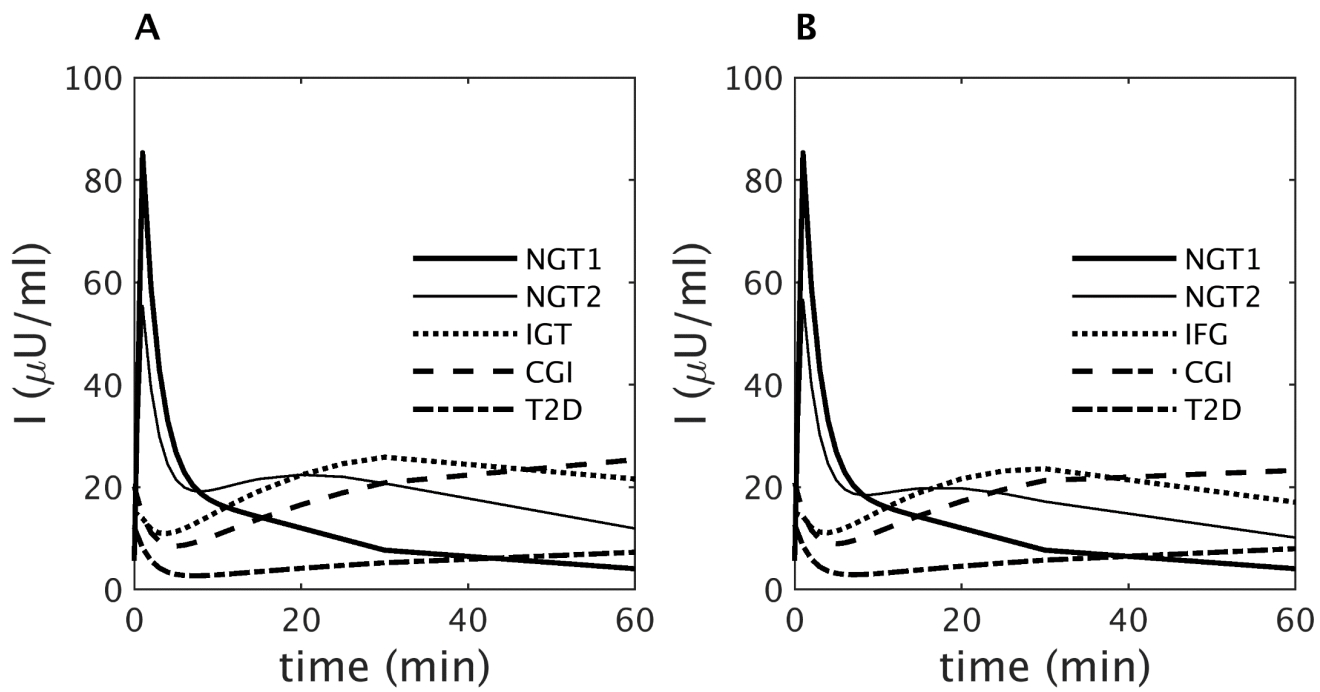

C
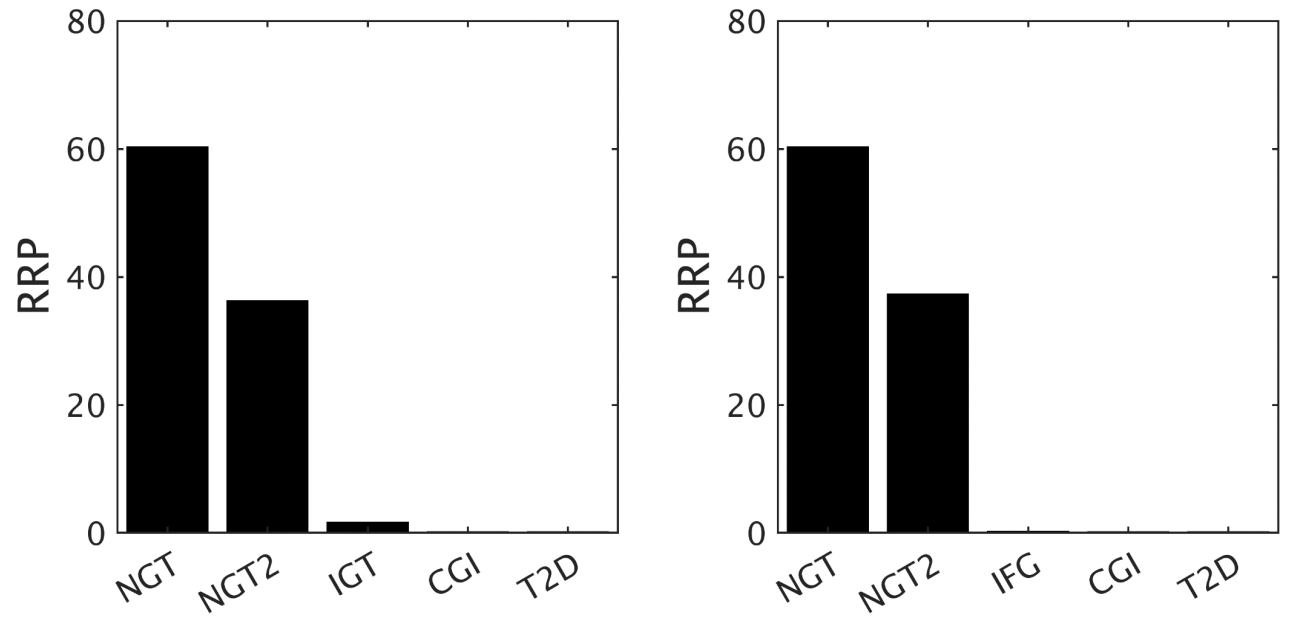


\section{Fig. 8}

A

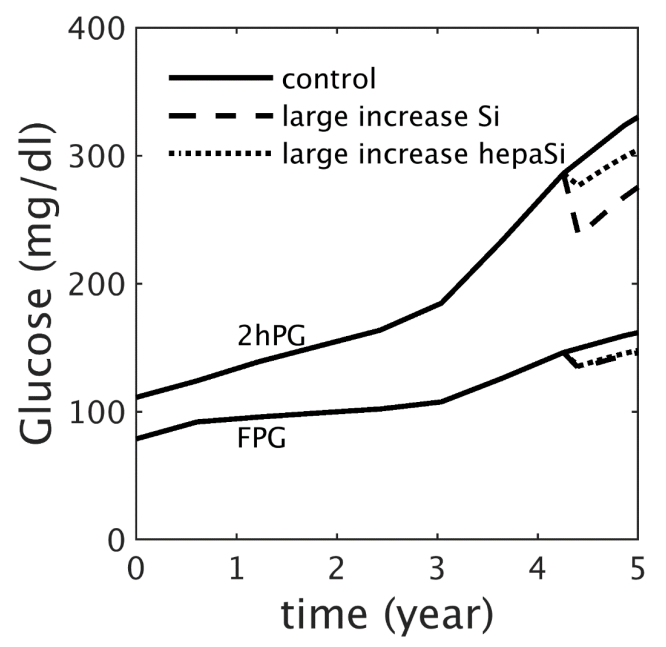

C

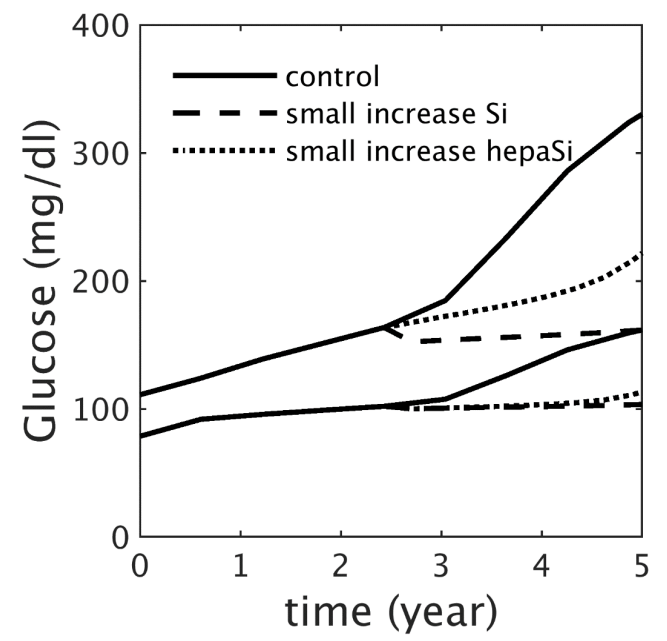

B

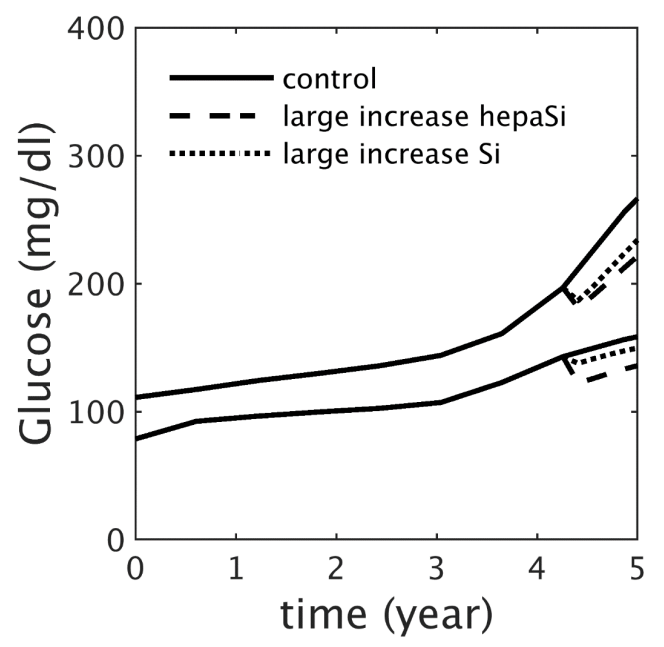

D

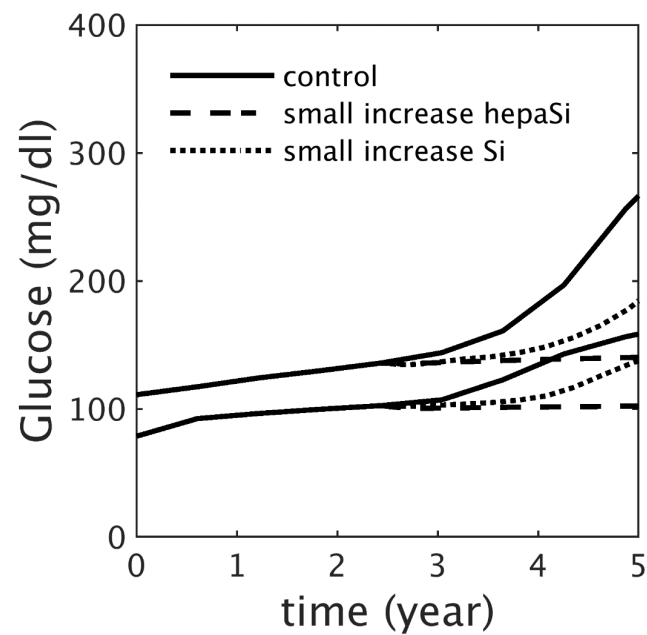


Fig. 9
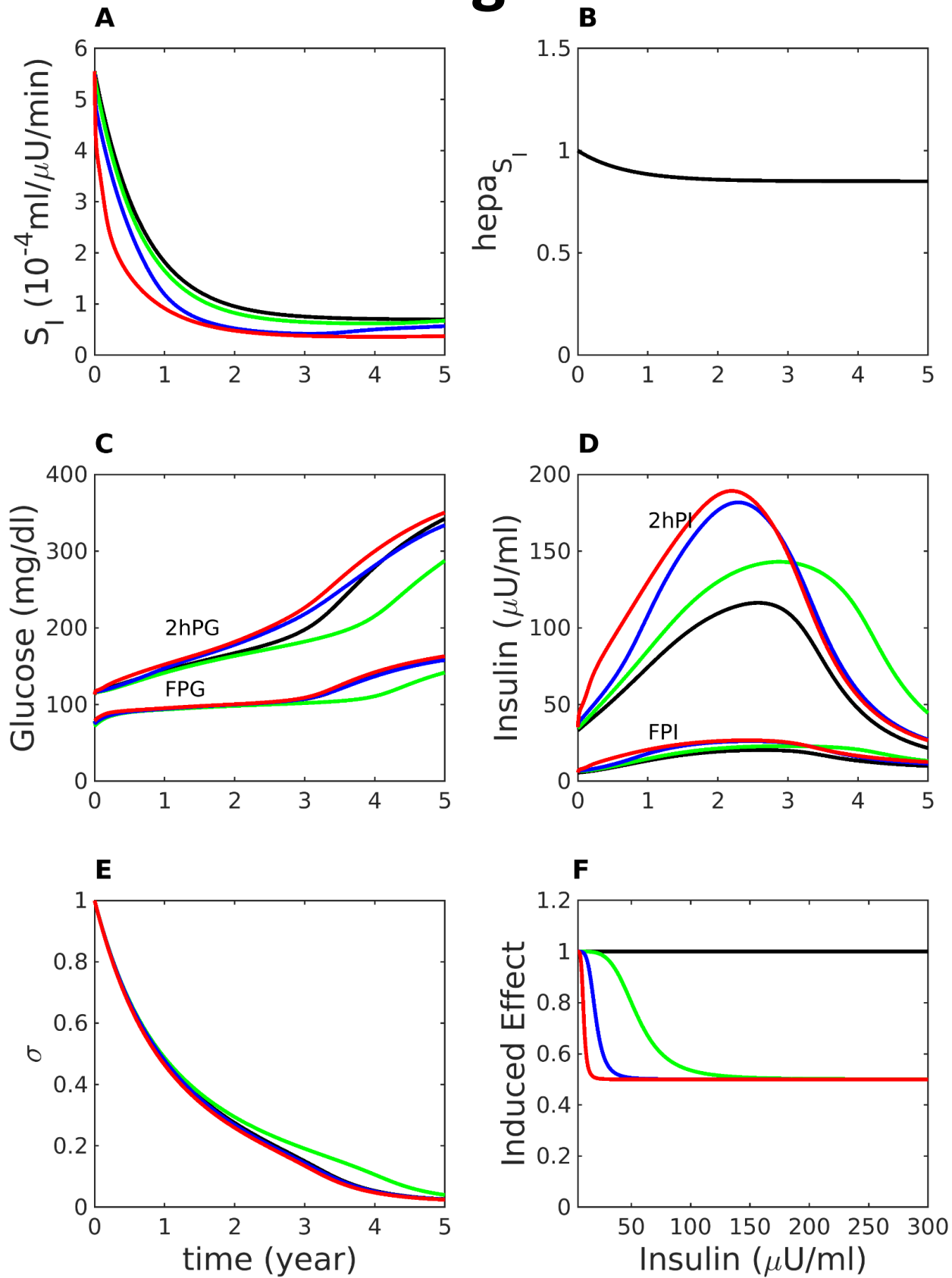